\title{
Section 4.7
}

\section{Further Topics in Connectivity}

\author{
Camino Balbuena, Universitat Politècnica de Catalunya, Spain \\ Josep Fàbrega, Universitat Politècnica de Catalunya, Spain \\ Miquel Àngel Fiol, Universitat Politècnica de Catalunya, Spain
}

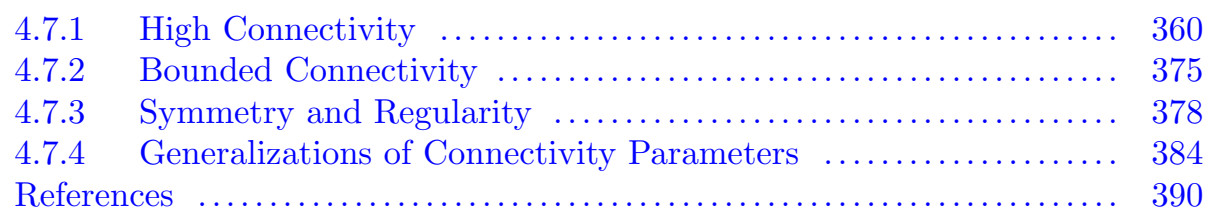

\section{INTRODUCTION}

Continuing the study of connectivity, initiated in $\S 4.1$ of the Handbook, we survey here some (sufficient) conditions under which a graph or digraph has a given connectivity or edge-connectivity. First, we describe results concerning maximal (vertex- or edge-) connectivity. Next, we deal with conditions for having (usually lower) bounds for the connectivity parameters. Finally, some other general connectivity measures, such as one instance of the so-called "conditional connectivity," are considered.

For unexplained terminology concerning connectivity, see $\S 4.1$.

\subsubsection{High Connectivity}

Since connectivity has to do with "connection," intuitively we can expect to find high connectivity when the "edge density" of the graph is large. Different situations in which this seems to be the case are:

(a) Large minimum or average degree.

(b) Small diameter (for given girth).

(c) Small number of vertices (for given degree and girth).

(d) Large number of vertices (for given degree and diameter).

The results in this subsection give several conditions of the above types, under which maximum vertex- or edge-connectivity is attained. An extensive collection of results about maximally edge-connected and vertex-connected graphs and digraphs can be found in the survey by Hellwig and Volkmann [HeVo08b]. 


\section{Minimum Degree and Diameter}

NOTATION: Let $G=(V, E)$ be a graph with order $n$, minimum degree $\delta$, maximum degree $\Delta$, edge-connectivity $\lambda$, and (vertex-)connectivity $\kappa$. In some other sections of the Handbook, the notations $\delta_{\min }, \delta_{\max }, \kappa_{e}$, and $\kappa_{v}$ are used instead of $\delta, \Delta, \lambda$, and $\kappa$, respectively.

\section{DEFINITIONS}

D1: The girth $g$ of a graph $G$ with a cycle is the length of its shortest cycle. An acyclic graph has infinite girth.

D2: The diameter $D$ of $G$ is $\max _{u, v \in V}\left\{\operatorname{dist}_{G}(u, v)\right\}$.

D3: The clique number of a graph $G$, denoted $\omega(G)$, is the maximum number of vertices in a complete subgraph of $G$.

D4: A (di)graph $G$ is $p$-partite if its vertex-set can be partitioned into $p$ independent (or stable) sets.

\section{FACTS}

F1: [Ch66] If $\delta \geq\lfloor n / 2\rfloor$, then $G$ is maximally edge-connected (i.e., $\lambda=\delta$ ).

F2: [Le74] If for any non-adjacent vertices $u$ and $v, \operatorname{deg}(u)+\operatorname{deg}(v) \geq n-1$, then $\lambda=\delta$.

F3: $[\mathrm{Pl75}]$ If $G$ is a graph with diameter $D=2$, then $\lambda=\delta$.

F4: $[\mathrm{HeVo08a}]$ For any graph $G, \lambda(G)=\delta(G)$ or $\lambda(\bar{G})=\delta(\bar{G})$.

F5: [Vo88] If $G$ is bipartite and $\delta \geq\lfloor n / 4\rfloor+1$, then $\lambda=\delta$.

F6: [Vo89] If $G$ is $p$-partite $(p \geq 2)$ and $n \leq 2\left\lfloor\frac{p}{p-1} \delta\right\rfloor-1$, then $\lambda=\delta$.

F7: [ToVo93] If $G$ is $p$-partite $(p \geq 2)$ and $\delta \geq n \frac{2 p-3}{2 p-1}$, then $G$ is maximally connected (i.e., $\kappa=\delta$ ).

F8: [DaVo95] If $G$ is $p$-partite $(p \geq 2)$ with clique number $\omega \leq p$ and $n \leq 2\left\lfloor\frac{p}{p-1} \delta\right\rfloor-1$, then $\lambda=\delta$.

\section{REMARKS}

R1: It is easily shown that Fact F3 $\Rightarrow$ Fact F2 $\Rightarrow$ Fact F1.

R2: Fact F5 is a slight improvement of Fact F6 for $p=2$.

R3: In addition to Fact F8, the authors in [DaVo95] gave other sufficient conditions for $\lambda=\delta$ that mostly generalize conditions in [PlZn89].

R4: A consequence of Fact F4 is that $\lambda(G)=\delta(G)$ for any self-complementary graph $(G=\bar{G})$. 


\section{Degree Sequence}

NOTATION: For the next group of results, $G$ is an $n$-vertex graph with degree sequence $d_{1} \geq d_{2} \geq \cdots \geq d_{n}=\delta$. For a vertex $u, N(u)$ denotes the set of vertices adjacent to $u$.

\section{FACTS}

F9: [GoWh78] If the vertex set of $G$ can be partitioned into $\lfloor n / 2\rfloor$ pairs of vertices $\left(u_{i}, v_{i}\right)$ (and, if $n$ is odd, one "unpaired" vertex $w$ ) such that $\operatorname{deg}\left(u_{i}\right)+\operatorname{deg}\left(v_{i}\right) \geq n$, $i=1,2, \ldots,\lfloor n / 2\rfloor$, then $\lambda=\delta$.

F10: [GoEn79] If each vertex $u$ of minimum degree satisfies

$$
\sum_{v \in N(u)} \operatorname{deg}(v) \geq \begin{cases}\lfloor n / 2\rfloor^{2}-\lfloor n / 2\rfloor, & \text { for even } n \text { or odd } n \leq 15 \\ \lfloor n / 2\rfloor^{2}-7, & \text { for odd } n \geq 15\end{cases}
$$

then $\lambda=\delta$.

F11: [Bo79] Let $G$ be a graph with order $n \geq 2$. If its degree sequence $d_{1} \geq d_{2} \geq \cdots \geq$ $d_{n}=\delta$ satisfies $\sum_{i=1}^{k}\left(d_{i}+d_{n-i}\right) \geq k n-1$ for all $k$ with $1 \leq k \leq \min \{\lfloor n / 2\rfloor-1, \delta\}$, then $\lambda=\delta$.

F12: [DaVo97] If $\delta \geq\lfloor n / 2\rfloor$ or if $\delta \leq\lfloor n / 2\rfloor-1$ and $\sum_{i=1}^{k}\left(d_{i}+d_{n+i-\delta-1}\right) \geq k(n-2)+$ $2 \delta-1$ for some $k$ with $1 \leq k \leq \delta$, then $\lambda=\delta$.

F13: [Vo03] Suppose that $G$ is $p$-partite $(p \geq 2)$ and has order $n \geq 6$ with clique number $\omega \leq p$. Let $\nu=1$ when $n$ is even and $\nu=0$ when $n$ is odd. If $\delta \geq\lfloor n / 2\rfloor$ or if $\delta \leq\lfloor n / 2\rfloor-1$ and $\sum_{i=1}^{\delta+1} d_{n+1-i} \geq(\delta+1) \frac{p-1}{p} \frac{n+1+\nu}{2}-\frac{2 \delta+2}{p(n-3+\nu)}$, then $\lambda=\delta$.

\section{REMARKS}

R5: Note that Fact F9 implies Fact F1 only when $n$ is even. Fact F10 also implies Fact F1. Moreover, as shown by the examples in [P1Zn89], Fact F10 is independent of Fact F2 and Fact F3.

R6: Fact F11 implies Fact F1 when $n$ is even, but in general, as shown in [PlZn89], it is independent of Facts 1, 2, 3 and 10.

R7: Fact F12 is even valid for digraphs, and a theorem of Xu [Xu94] follows easily (see Fact F23). It is easily shown that Fact F12 implies Fact F11.

R8: Fact F13 generalizes results in [Vo88, Vo89], as well as Fact F8. Furthermore, as shown in [HeVo03b], the conditions in Fact F13 also guarantee maximum local edgeconnectivity for all pairs $u$ and $v$ of vertices in $G$; that is, $\lambda(u-v)=\min \{\operatorname{deg}(u), \operatorname{deg}(v)\}$. 


\section{Distance}

\section{DEFINITIONS}

D5: The distance $\operatorname{dist}_{G}\left(U_{1}, U_{2}\right)$ between two given subsets $U_{1}, U_{2} \subset V(G)$ is the minimum of the distances $\operatorname{dist}_{G}\left(u_{1}, u_{2}\right)$ for all vertices $u_{1} \in U_{1}$ and $u_{2} \in U_{2}$. (When there is no ambiguity, we omit the subscript $G$.)

D6: The line graph $L(G)$ of a graph $G$ has vertices representing the edges of $G$, and two vertices are adjacent if and only if the corresponding edges are adjacent (that is, they have one endpoint in common).

\section{FACTS}

F14: Let $u_{1} v_{1}$ and $u_{2} v_{2}$ be edges in a graph $G$, and let $U_{i}=\left\{u_{i}, v_{i}\right\}, i=1,2$. Then, the distance between the corresponding vertices of $L(G)$ satisfies $d_{L(G)}\left(u_{1} v_{1}, u_{2} v_{2}\right)=$ $d_{G}\left(U_{1}, U_{2}\right)+1$ and thus, the diameters of $L(G)$ and $G$ satisfy $D(L(G)) \leq D(G)+1$.

F15: $[\mathrm{PlZn} 89]$ Let $G$ be a connected graph such that every pair of vertex subsets $U_{1}, U_{2}$ of cardinality two satisfies $\operatorname{dist}\left(U_{1}, U_{2}\right) \leq 2$. Then $\lambda=\delta$.

F16: $[\mathrm{BaCaFaFi96}]$ Let $G$ be a graph with minimum degree $\delta$ and line graph $L(G)$. Then,

(a) If $L(G)$ has diameter at most three, then $\lambda=\delta$.

(b) If $L(G)$ has diameter two, then $\kappa=\delta$.

\section{REMARKS}

R9: The sufficient condition given in Fact F15 is slightly weaker than the one given in Fact F3. Furthermore, it suffices to require such a condition on the 2-element subsets that are the endpoints of some edge, as shown in Fact F16(a).

R10: From the above remark, Fact F16(a) generalizes both Fact F15 and Fact F3 (Plesnik's result).

\section{Super Edge-Connectivity}

Here we consider a stronger measure of edge-connectivity.

\section{DEFINITION}

D7: A maximally edge-connected graph is super- $\lambda$ if every minimum edge-disconnecting set is trivial, that is, consists of the edges incident on a vertex of minimum degree. 


\section{EXAMPLE}

E1: $\quad$ Figure 4.7.1 shows a 3-regular maximally edge-connected graph that is not super- $\lambda$. The set $\{e, f, g\}$ is a non-trivial minimum edge-disconnecting set.

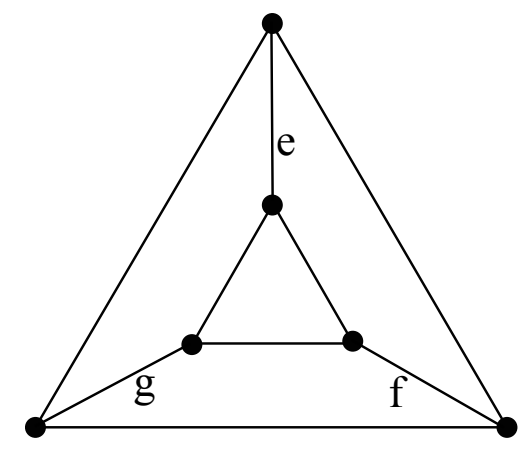

Figure 4.7.1: $G$ is maximally edge-connected but not super- $\lambda$.

\section{FACTS}

F17: [Le74] Let $G \neq K_{n / 2} \times K_{2}$. If for any non-adjacent vertices $u$ and $v, \operatorname{deg}(u)+$ $\operatorname{deg}(v) \geq n$, then $G$ is super- $\lambda$.

F18: If for any non-adjacent vertices $u$ and $v, \operatorname{deg}(u)+\operatorname{deg}(v) \geq n+1$, then $G$ is super- $\lambda$.

F19: $[\operatorname{Ke} 72]$ If $\delta \geq\lfloor n / 2\rfloor+1$, then $G$ is super- $\lambda$.

F20: [Fi92] If $G$ has diameter two and contains no complete subgraph $H$ on $\delta$ vertices with $\operatorname{deg}_{G}(v)=\delta$ for all $v \in V(H)$, then $G$ is super- $\lambda$.

F21: [So92] Let $G$ be a graph with maximum degree $\Delta$. If $n>2 \delta+\Delta-1$, then $G$ is super- $\lambda$.

\section{REMARKS}

R11: Facts F18 and F19, which are analogues of Facts F2 and F1, are direct consequences of Fact F17.

R12: Fact F20 can be seen as a refinement of Fact F3 (where only the diameter condition is required) and has Fact F21 as a corollary.

\section{Digraphs}

As mentioned in $\S 4.1$, since the connectivity parameters of a graph $G$ equal those of its symmetric digraph $G^{*}$ (obtained by replacing each edge of $G$ by a digon), many of the previous results can be generalized to the directed case.

\section{DEFINITIONS}

D8: The vertex-connectivity of a digraph $G$, denoted $\kappa(G)$, is the minimum size of a vertex subset whose deletion results in a non-strongly connected or trivial digraph. 
D9: The edge-connectivity of a digraph $G$, denoted $\lambda(G)$, is the minimum size of an edge subset whose deletion results in a non-strongly connected digraph.

notation: (a) For a vertex $u \in V(G), \operatorname{deg}^{+}(u)$ denotes the out-degree, the number of vertices adjacent from vertex $u$, and $\operatorname{deg}^{-}(u)$ denotes the in-degree, the number of vertices adjacent to vertex $u$. Let $\delta(u)=\min \left\{\operatorname{deg}^{+}(u), \operatorname{deg}^{-}(u)\right\}$.

(b) $\delta^{+}=\min _{u \in V}\left\{\operatorname{deg}^{+}(u)\right\}$ and $\delta^{-}=\min _{u \in V}\left\{\operatorname{deg}^{-}(u)\right\}$.

(c) $\delta=\min _{u \in V} \delta(u)=\min \left\{\delta^{+}, \delta^{-}\right\}$.

Similar notations with $\Delta$ stand for maximum degrees.

NOTATION: For vertices $u, v \in V(G), \lambda(u-v)$ denotes the maximum number of edgedisjoint directed paths from $u$ to $v$.

\section{FACTS}

F22: [Jo72] If $G$ is a digraph with diameter $D=2$, then $\lambda=\delta$.

F23: [Xu94] Let $G$ be a digraph of order $n$. If there are $\lfloor n / 2\rfloor$ pairs of (different) vertices $\left(u_{i}, v_{i}\right)$ such that $\delta\left(u_{i}\right)+\delta\left(v_{i}\right) \geq n, i=1,2, \ldots,\lfloor n / 2\rfloor$, then $\lambda=\delta$.

F24: [HeVo03b] Let $G$ be a digraph with diameter at most two. Then, $\lambda(u-v)=$ $\min \left\{\operatorname{deg}^{+}(u), \operatorname{deg}^{-}(v)\right\}$ for all pairs $u$ and $v$ of vertices in $G$.

F25: [HeVo03a] Let $G$ be a strongly connected digraph with edge-connectivity $\lambda$ and minimum degree $\delta$. If for all maximal pairs of vertex sets $X$ and $Y$ at distance 3 there exists an isolated vertex in the induced subgraph on $X \cup Y$, then $\lambda=\delta$.

F26: $[\mathrm{HeVo03b}$ Let $G$ be a $p$-partite digraph of order $n$ and minimum degree $\delta$ with $p \geq 2$. If $n \leq 2\lfloor(p \delta) /(p-1)\rfloor-1$, then $\lambda(u-v)=\min \left\{\operatorname{deg}^{+}(u), \operatorname{deg}^{-}(v)\right\}$ for all pairs $u$ and $v$ of vertices in $G$.

F27: $[\mathrm{HeVo03b]}$ Let $G$ be a bipartite digraph of order $n$ and minimum degree $\delta \geq 2$ with the bipartition $V^{\prime} \cup V^{\prime \prime}$. If $\operatorname{deg}(x)+\operatorname{deg}(y) \geq(n+1) / 2$ for each pair of vertices $x, y \in V^{\prime}$ and each pair of vertices $x, y \in V^{\prime \prime}$, then $\lambda(u-v)=\min \left\{\operatorname{deg}^{+}(u), \operatorname{deg}^{-}(v)\right\}$ for all pairs $u$ and $v$ of vertices in $G$.

\section{REMARKS}

R13: Notice that Plesník's result (Fact F3) is, in fact, a consequence of the older result of Jolivet (Fact F22). Similarly, Fact F23 generalizes Fact F9.

R14: Fact F23 was improved by Dankelmann and Volkmann in two subsequent papers [DaVo97, DaVo00], where the bipartite case was also considered.

R15: A restatement of Fact F24 states that a digraph with diameter two has maximum local edge-connectivity. Moreover, this obviously implies Jolivet's result (Fact F22) and the corresponding local connectivity result for undirected graphs, proved in [FrOeSw00].

R16: A consequence of Fact F25 is the directed version of Fact F15. 


\section{Oriented Graphs}

\section{DEFINITIONS}

D10: A digraph is super- $\lambda$ if every minimum edge-disconnecting set consists of the edges directed to or from a vertex with minimum degree. A digraph is super- $\kappa$ if every minimum disconnecting set consists of the vertices adjacent to or from a vertex with minimum degree.

D11: An oriented graph $G$ (also called an antisymmetric digraph) is a digraph such that between any two vertices $u, v$, there is at most one (directed) edge $((u, v)$ or $(v, u))$.

\section{EXAMPLE}

E2: Figure 4.7.2 shows a 2-regular maximally connected digraph $G$ that is not super- $\kappa$. If $F=\{x, y\}$, then $G-F$ is not strongly connected (for instance, there is no [directed] path in $G-F$ from $u$ to $v$ ) and $F$ is non-trivial (it does not consist of the vertices adjacent to or from a vertex with minimum degree).

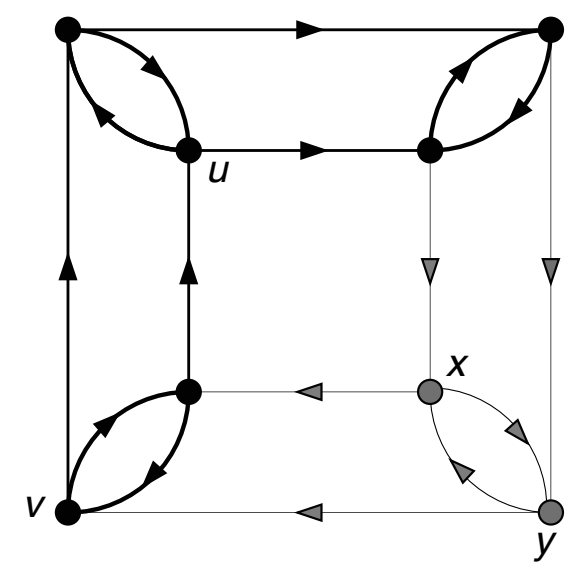

Figure 4.7.2: $G$ is maximally connected but not super- $\kappa$.

\section{FACTS}

F28: [AyFr70] Let $G$ be an oriented graph with $n$ vertices and minimum degree $\delta$. If $\delta \geq\lfloor(n+2) / 4\rfloor$, then $\lambda=\delta$.

F29: [Fi92] If $G$ is an oriented graph with $n$ vertices and minimum degree $\delta \geq\lfloor n / 4\rfloor+1$, then $G$ is super- $\lambda$.

F30: [Fi92] If $G$ is an oriented graph with diameter two, then $G$ is super- $\lambda$.

\section{REMARKS}

R17: Facts F28 and F29 are analogues of Fact F1, whereas Fact F30, similar to Fact F22, is a consequence of Fact F20. 
R18: In fact, the sufficient conditions given in [AyFr70] and [Fi92] (Facts F28 and F29) were $\delta^{+}+\delta^{-} \geq\lfloor n / 2\rfloor$ and $\delta^{+}+\delta^{-} \geq\lfloor n / 2\rfloor+1$, respectively. Furthermore, it is easily shown that Facts F29 and F30 do not imply each other.

R19: Higher connectivity in tournaments, which are oriented complete graphs, is discussed in $\S 3.3$ of the Handbook.

\section{Semigirth}

To generalize Jolivet's result (Fact F22) and give new results on superconnectivity, it is relevant to consider a new parameter related to the path structure of the digraph. In our context, this parameter plays a role similar (and is tightly related) to the girth of a graph.

\section{DEFINITIONS}

D12: [FaFi89, FiFaEs90] For a given digraph $G=(V, E)$ with diameter $D$, the semigirth, denoted $\ell(G)$, is the greatest integer $\ell$ between 1 and $D$ such that for any $u, v \in V$,

(a) if $\operatorname{dist}(u, v)<\ell$, the shortest $u-v$ directed walk is unique and there are no $u-v$ directed walks of length $\operatorname{dist}(u, v)+1$.

(b) if $\operatorname{dist}(u, v)=\ell$, there is only one shortest $u$-v directed walk.

D13: A digraph $G$ is a generalized p-cycle when it has its vertex set partitioned in $p$ parts cyclically ordered, and vertices in one part are adjacent only to vertices in the next part. Thus, a generalized 2-cycle is the same as a bipartite digraph.

\section{EXAMPLE}

E3: Figure 4.7.3 shows a 2-regular digraph for which the semigirth $\ell$ is equal to its diameter, namely, $\ell=D=3$.

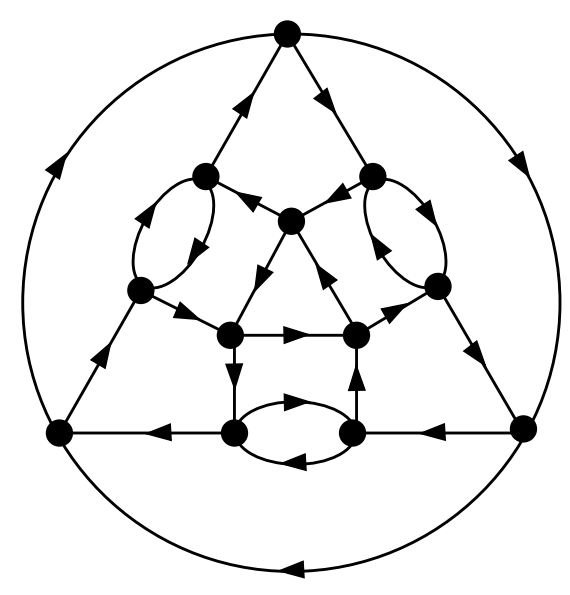

Figure 4.7.3: Semigirth $\ell=D=3$. 


\section{FACTS}

F31: [FaFi89] Let $G$ be a digraph with minimum degree $\delta>1$, diameter $D$, semigirth $\ell$, and connectivities $\kappa$ and $\lambda$.

(a) If $D \leq 2 \ell$, then $\lambda=\delta$.

(b) If $D \leq 2 \ell-1$, then $G$ is super- $\lambda$ and $\kappa=\delta$.

(c) If $D \leq 2 \ell-2$, then $G$ is super- $\kappa$.

F32: $\quad[$ FaFi96a, PeBaGo01] Let $G$ be a generalized $p$-cycle $(p \geq 2)$.

(a) If $D \leq 2 \ell+p-1$, then $\lambda=\delta$.

(b) If $D \leq 2 \ell+p-2$, then $G$ is super- $\lambda$ and $\kappa=\delta$.

(c) If $D \leq 2 \ell+p-3$, then $G$ is super- $\kappa$.

F33: Any bipartite digraph with diameter three is maximally edge-connected.

\section{REMARKS}

R20: The main idea in the proof of the results in Fact F31 is that semigirth $\ell$ measures how far away one can move from or to a given subset $F$ of vertices. For instance, in proving (a), it is shown that if $|F|<\delta$, in any connected component of $G-F$ there are vertices $u, v$ such that $\operatorname{dist}(u, F), \operatorname{dist}(F, v) \geq \ell$. Hence, any shortest path of length at most $2 \ell-1$ cannot contain a vertex of $F$. As a conclusion, $F$ cannot be a disconnecting set.

R21: Since any digraph $G$ has semigirth $\ell \geq 1$, Fact F22 is included in Fact F31(a).

R22: Fact F33 is the analogue for bipartite digraphs of Jolivet's result (Fact F22). In fact, for a bipartite (di)graph, the condition $\delta \geq\lfloor n / 4\rfloor+1$ implies $D \leq 3$, so that Fact F33 can be also seen as a generalization of Fact F5.

\section{Line Digraphs}

\section{DEFINITION}

D14: The line digraph of a digraph $G$, denoted $L(G)$, has $V(L(G)) \equiv E(G)$, and a vertex $(u, v)$ is adjacent to a vertex $(w, z)$ if $v=w$ (that is, the head of edge $(u, v)$ is the tail of edge $(w, z)$ in digraph $G)$. The $k$-iterated line digraph, $L^{k}(G)$, is defined recursively by $L^{k}(G)=L\left(L^{k-1}(G)\right)$.

\section{FACTS}

F34: The order of $L(G)$ equals the size of $G,|V(L(G))|=|E(G)|$, and their minimum degrees coincide, $\delta(L(G))=\delta(G)=\delta$. Moreover, $\kappa(L(G))=\lambda(G)$. 
F35: If $G$ is $d$-regular, $d>1$, has order $n$, diameter $D$, and semigirth $\ell$, then $L^{k}(G)$ is also $d$-regular, has $d^{k} n$ vertices, diameter $D\left(L^{k}(G)\right)=D(G)+k$, and semigirth $\ell\left(L^{k}(G)\right)=\ell(G)+k$. See the papers [Ai67, ReKuHoLe82, FiYeAl84, FaFi89].

F36: $[\mathrm{FaFi} 89]$ Let $G$ be a digraph with minimum degree $\delta>1$, diameter $D$, and semigirth $\ell$.

(a) If $k \geq D-2 \ell$, then $L^{k}(G)$ is maximally edge-connected.

(b) If $k \geq D-2 \ell+1$, then $L^{k}(G)$ is super- $\lambda$ and maximally connected.

(c) If $k \geq D-2 \ell+2$, then $L^{k}(G)$ is super- $\kappa$.

\section{REMARK}

R23: As shown in Fact F36, the interest of considering $k$-iterated line digraphs stems from the fact that if $k$ is large enough, Fact F35 guarantees that the conditions of Fact F31 hold.

\section{Girth}

For a given girth, high density/connectivity graphs occur when they have a reduced diameter, and also when they have a small number of vertices.

\section{DEFINITION}

D15: The same definition for the semigirth (Definition D12) applies for an undirected graph $G$ (considering undirected walks). In this case, it turns out that the semigirth $\ell=\ell(G)=\ell\left(G^{*}\right)$ equals $\lfloor(g-1) / 2\rfloor$ where $g=g(G)$ stands for the girth of $G$.

\section{FACTS}

F37: Let $G$ be a graph with minimum degree $\delta>1$, diameter $D$, girth $g$, and connectivities $\kappa$ and $\lambda$.

(a) [SoNaIm85, SoNaImPe87, FaFi89] If $D \leq\left\{\begin{array}{ll}g-1, & g \text { odd, } \\ g-2, & g \text { even, }\end{array}\right.$ then $\lambda=\delta$.

(b) [SoNaIm85, SoNaImPe87, FaFi89] If $D \leq\left\{\begin{array}{ll}g-2, & g \text { odd, } \\ g-3, & g \text { even, }\end{array}\right.$ then $G$ is super- $\lambda$ and $\kappa=\delta$.

(c) [SoNaIm85, SoNaImPe87, FaFi89] If $D \leq\left\{\begin{array}{ll}g-3, & g \text { odd, } \\ g-4, & g \text { even, }\end{array}\right.$ then $G$ is super- $\kappa$.

(d) $[\mathrm{BaCeDiGVMa06]}$ If $D \leq g-3$, then $G$ is super- $\kappa$.

(e) [BaTaMaLi09] If $G$ is regular and $D \leq g-2, g$ odd, then $G$ is super- $\kappa$.

(f) [BaMaMo10] If $\delta \geq 3, \Delta \leq 3 \delta / 2-1$, and $D \leq g-2, g$ odd, then $G$ is super- $\kappa$. 
F38: [BaCaFaFi96, CaFa99] Let $G$ be a graph with minimum degree $\delta>1$, girth $g$, and connectivities $\kappa$ and $\lambda$. Let $L(G)$ be the line graph of $G$, with diameter $D(L(G))$. Then,
(a) If $D(L(G)) \leq\left\{\begin{array}{ll}g, & g \text { odd, } \\ g-1, & g \text { even, }\end{array}\right.$ then $\lambda=\delta$.
(b) If $D(L(G)) \leq\left\{\begin{array}{ll}g-1, & g \text { odd, } \\ g-2, & g \text { even, }\end{array}\right.$ then $G$ is super- $\lambda$ and $\kappa=\delta$.
(c) If $D(L(G)) \leq\left\{\begin{array}{ll}g-2, & g \text { odd, } \\ g-3, & g \text { even, }\end{array}\right.$ then $G$ is super- $\kappa$.

F39: [FaFi96a] Any bipartite graph with diameter three is maximally edge-connected.

F40: [KnNi03] For every graph $G$ there is a number $i(G)$ such that $L^{k}(G)$ is maximally connected when $k \geq i(G)$.

\section{REMARKS}

R24: Fact F37 is a simple consequence of Definition D15 and Fact F31.

R25: Fact F39 is the undirected version of Fact F33, which can be seen as Plesník's analogue for the bipartite case.

R26: Fact F40 is based on a result of Hartke and Higgins [HaHi99] about the growth of minimum degree in iterated line graphs. For regular graphs this result is not needed, and in this case $i(G) \leq 5$.

\section{Girth Pair}

\section{DEFINITIONS}

D16: The girth pair $\left(g_{1}, g_{2}\right)$ of a graph $G$ gives the length $g_{1}$ of a shortest odd cycle and the length $g_{2}$ of a shortest even cycle.

\section{EXAMPLE}

E4: The Dodecahedron graph is a cubic graph with girth 5 and a shortest even cycle has length 8 . Hence its girth pair is $(5,8)$.

\section{FACTS}

F41: [BaCeDiGVMa07, BaGVMo11] Let $G$ be a graph with minimum degree $\delta \geq 3$, diameter $D$, girth pair $(g, h)$, odd $g$ and even $h$ with $g+3 \leq h<\infty$, and connectivities $\kappa$ and $\lambda$.

(a) If $D \leq h-3$, then $\lambda=\delta$.

(b) If $D \leq h-4$, then $\kappa=\delta$. 
(c) If $D \leq h-4$ and $\delta \geq 4$, then $G$ is super- $\kappa$.

(d) If $D \leq h-5$ and $\delta=3$, then $G$ is super- $\kappa$.

(e) If $g \geq 5, D(L(G)) \leq h-3$, and the maximum degree of $G$ satisfies $\Delta \leq 2 \delta-3$, then $\kappa=\delta$.

\section{REMARKS}

R27: Fact F41 improves Fact F37 for graphs with girth pair $(g, h), g$ odd and $h \geq g+3$ even.

\section{Cages}

\section{DEFINITIONS}

D17: A $(k, g)$-cage is a $k$-regular graph with girth $g$ having the least possible number of vertices.

D18: A 3-connected graph $G=(V, E)$ is said to be quasi 4-connected if for every vertex-cut $F \subset V$ such that $|F|=3, F$ is the neighborhood of a vertex of degree 3 and $G-F$ has exactly two components.

\section{EXAMPLE}

E5: The Heawood graph, shown in Figure 4.7.4, is a $(3,6)$-cage with order 14 and diameter 3 .

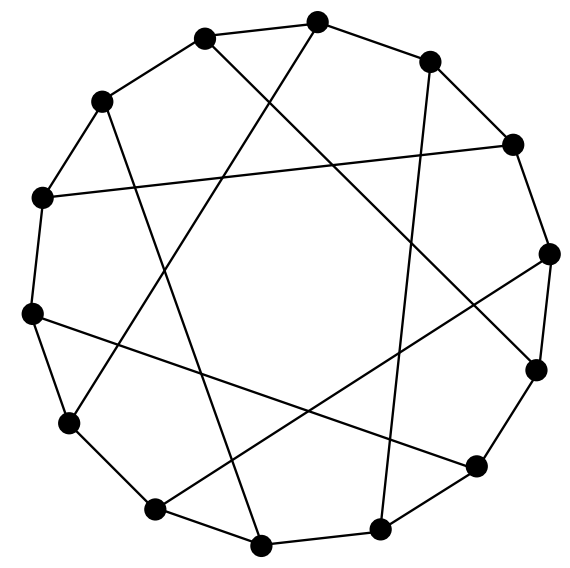

Figure 4.7.4: The Heawood graph.

\section{FACTS}

F42: [FuHuRo97] All $(k, g)$-cages are 2-connected.

F43: [JiMu98, DaRo99] Every $(k, g)$-cage with $k \geq 3$ is 3 -connected. 
F44: [MaPeBa02] Every $(3, g)$-cage is superconnected, edge-superconnected, and quasi 4-connected.

F45: [WaXuWa03] Every $(k, g)$-cage with $k \geq 3$ and odd girth $g$ is maximally edgeconnected.

F46: $[\mathrm{MaBa04}]$ Every $(k, g)$-cage with $k \geq 3$ and odd girth $g$ is super- $\lambda$.

F47: $[$ LiMiBaMa06] Every $(k, g)$-cage with $k \geq 3$ and even girth $g$ is super- $\lambda$.

F48: [XuWaWa02] Every $(4, g)$-cage is 4-connected.

F49: $[\mathrm{MaBaPeFa} 05]$ Every $(k, g)$-cage with $k \geq 4$ and $g \geq 10$ is 4-connected.

F50: [LiMiBa05] Every $(k, g)$-cage with $k \geq 3$ and odd girth $g \geq 7$ is $r$-connected with $r \geq \sqrt{k+1}$.

F51: [LiBaMaMi08] Every $(k, g)$-cage with $k \geq 3$ and even girth $g \geq 6$ is $(r+1)$ connected, $r$ being the largest integer such that $r^{3}+2 r^{2} \leq k$.

F52: $[\mathrm{MaBaPe} 07](k, 6)$ - and $(k, 8)$-cages are maximally connected.

F53: [ArGoMoSe07] $(k, 12)$-cages are maximally connected.

F54: [LuWuLuLi10] Every $(k, g)$-cage with $k \geq 3$ and odd girth $g \geq 9$ is $\lceil\sqrt{k+\sqrt{k}-2}\rceil$ connected; and every $(k, g)$-cage with $k \geq 3$ and even girth $g \geq 10$ is $r$-connected, where $r$ is the largest integer such that $r(r-1)^{2} / 4+1+2 r(r-1) \leq k$.

F55: [BaSa12] Every $(k, g)$-cage with $k \geq 3$ and odd girth $g \geq 7$ is $r$-connected with $r \geq\lceil k / 2\rceil$.

\section{CONJECTURE}

[FuHuRo97] Every $(k, g)$-cage is maximally connected.

\section{Large Digraphs}

The following results support the intuitive idea that dense (di)graphs have high connectedness.

\section{DEFINITION}

D19: For a digraph with maximum degree $\Delta$ and diameter $D$, the Moore bound, denoted $n(\Delta, D)$, is given by $n(\Delta, D)=1+\Delta+\Delta^{2}+\cdots+\Delta^{D}$.

\section{FACTS}

F56: An $n$-vertex digraph with maximum degree $\Delta$ and diameter $D$ has $n \leq n(\Delta, D)$.

F57: [Wa67] The order of a (di)graph with connectivity $\kappa>1$ and diameter $D$ satisfies $n \geq \kappa(D-1)+2$.

F58: $[\operatorname{ImSoOk} 85]$

(a) If $\lambda<\delta$, then $n \leq \lambda(n(\Delta, D-2)+\Delta+1)$.

(b) If $\kappa<\delta$, then $n \leq \kappa(n(\Delta, D-1)+\Delta)$. 


\section{F59:}

(a) If $n>(\delta-1)(n(\Delta, D-2)+\Delta+1)$, then $\lambda=\delta$.

(b) If $n>(\delta-1)(n(\Delta, D-1)+\Delta)$, then $\kappa=\delta$.

F60: [Fi93]

(a) If $\lambda<\delta$, then $n \leq \lambda(n(\Delta, D-2)+1)+\Delta$.

(b) If $\kappa<\delta$, then $n \leq \kappa(n(\Delta, D-1)-1)+\Delta+1$.

F61: [Xu92, Fi93] Let $G$ be $d$-regular.

(a) If $n>d^{D-1}+2 d-2$, then $\lambda=d$.

(b) If $n>d^{D}+1$, then $\kappa=d$.

F62: $[$ So92, Fi94] Let $G$ be a $d$-regular digraph, $d \geq 2$, with diameter $D$.

(a) If $G$ satisfies either of the following conditions, then $G$ is super- $\lambda$.

(i) $D=2$ and $n>3 d$.

(ii) $D \geq 3$ and $n>2 d^{D-1}+d^{D-2}+\cdots+d^{2}+2 d$.

(b) If $G$ satisfies either of the following conditions, then $G$ is super- $\kappa$.

(i) $D=3$ and $n>3 d^{2}+1$.

(ii) $D \geq 4$ and $n>2 d^{D-1}+d^{D-2}+\cdots+d^{3}+2 d^{2}+1$.

\section{EXAMPLE}

E6: Figure 4.7.5 shows a regular digraph for which $n=6, \Delta=\delta=d=2$, and $D=2$. Since $n>d^{D-1}+2 d-2$ and $n>d^{D}+1$, Fact F61 guarantees that it is maximally connected $(\kappa=\lambda=d)$.

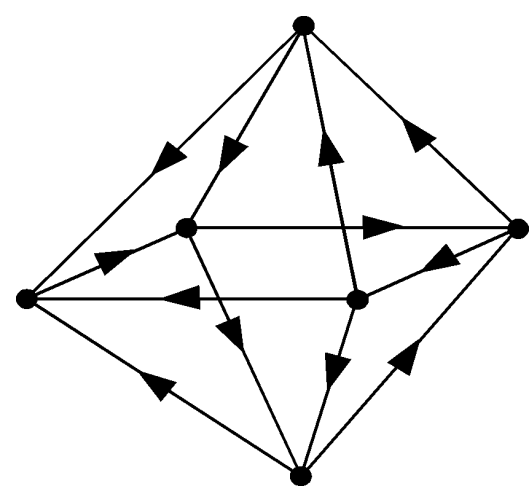

Figure 4.7.5: $\kappa=\lambda=d=2$. 


\section{REMARKS}

R28: To our knowledge, Fact F57, due to Watkins, was the first result in which the order $n$, the diameter $D$, and the connectivity $\kappa$ were related (in the undirected case). It follows easily from counting the minimum number of vertices involved in $\kappa$ internally disjoint $u-v$ paths between a pair of vertices $u, v$ at distance $D$, as Menger's theorem guarantees.

R29: Similar reasoning gives a lower bound for the number of edges $m$ of a (di)graph with edge-connectivity $\lambda$, namely, $m \geq \lambda D$. However, it is not difficult to realize that this is not a very strong result. (The situation seems to depend heavily on the values of $\lambda$ and $D$ : for $\lambda=3$ there are constructions giving a lower bound of the order of $\frac{7}{2} D$, whereas for $\lambda=4$ we have a bound which is "asymptotically optimal," that is, of the order of $4 D$.)

R30: If we take into account the connectivity parameters $\kappa$ or $\lambda$, the Moore bound can be refined. Intuitively, a disconnecting set with few vertices or edges is a kind of "bottleneck" that prevents the order from being large, as shown in Fact F58.

R31: Fact F59 is a direct consequence of Fact F58, and Fact F60 is an improvement of Fact F58. Notice that if we set $\kappa=\Delta$ in the upper bound on $n$ of Fact F60(b), we obtain the Moore bound $n(\Delta, D)$.

\section{Large Graphs}

Similar results for graphs were derived independently by Esfahanian [Es85], Fiol [Fi93, Fi94], and Soneoka et al. [SoNaImPe87].

\section{DEFINITION}

D20: The Moore bound for an undirected graph with maximum degree $\Delta$ and diameter $D$ is given by $n(\Delta, D)=1+\Delta+\Delta(\Delta-1)+\cdots+\Delta(\Delta-1)^{D-1}$.

\section{FACTS}

F63: [SoNaImPe87]

(a) If $n>(\delta-1)(n(\Delta-1, D-2)+1)+\Delta-1$, then $\lambda=\delta$.

(b) If $n>(\delta-1)(\Delta-1)^{D-1}+2$, then $\kappa=\delta$.

F64: [So92, Fi94]

(a) Let $D \geq 2$ and $\delta \geq 2$. If $n>\delta(n(\Delta-1, D-2)+1)+(\Delta-1)^{D-1}$, then $G$ is super- $\lambda$.

(b) Let $D \geq 3, \delta \geq 3$ and $g \geq 5$. If $n>(\delta-1)(n(\Delta, D-1)+\Delta)$, then $\kappa=\delta$. 


\subsubsection{Bounded Connectivity}

The techniques used for proving the results of the preceding subsection can often be used to derive bounds on the connectivity or edge-connectivity of a (di)graph. In this subsection, we provide some examples.

\section{$\pi$-Semigirth}

The following definition generalizes semigirth (Definition D12).

\section{DEFINITION}

D21: [FaFi89] Let $G=(V, E)$ be a digraph with minimum degree $\delta$ and diameter $D$, and let $\pi$ be an integer, $0 \leq \pi \leq \delta-2$. The $\pi$-semigirth of $G$, denoted $\ell_{\pi}(G)$, is the greatest integer $\ell_{\pi}$ between 1 and $D$ such that, for any $u, v \in V$,

(a) if $\operatorname{dist}(u, v)<\ell_{\pi}$, the shortest $u-v$ path is unique and there are at most $\pi$ distinct $u-v$ walks of length $\operatorname{dist}(u, v)+1$.

(b) if $\operatorname{dist}(u, v)=\ell_{\pi}$, there is only one shortest $u$ - $v$ walk.

\section{FACT}

F65: $[\mathrm{FaFi} 89$, MaBaPe04] Let $G$ be a connected digraph with minimum degree $\delta>1$, diameter $D, \pi$-semigirth $\ell_{\pi}$ for $0 \leq \pi \leq \delta-2$, and with $k$-iterated line $\operatorname{digraph} L^{k}(G)$. Then,

(a) If $D \leq 2 \ell_{\pi}$, then $\lambda \geq \delta-\pi$.

(b) If $D \leq 2 \ell_{\pi}-1$, then $\kappa \geq \delta-\pi$.

(c) If $D \leq 2 \ell_{\pi}-1$ and $\pi \leq\lfloor(\delta-1) / 2\rfloor$, then $\lambda=\delta$.

(d) If $D \leq 2 \ell_{\pi}-2, \ell_{0} \geq 2$ and $\pi \leq\lfloor(\delta-1) / 2\rfloor$, then $\kappa=\delta$.

(e) If $k \geq D-2 \ell_{\pi}$, then $\lambda\left(L^{k}(G)\right) \geq \delta-\pi$.

(f) If $k \geq D-2 \ell_{\pi}+1$, then $\kappa\left(L^{k}(G)\right) \geq \delta-\pi$.

\section{REMARKS}

R32: Note that $\ell_{0}$ corresponds to the ordinary semigirth $\ell$. Moreover, for $\pi \geq 1, \ell_{\pi}$ is well defined even for a digraph with self-loops.

R33: The definition of $\ell_{\pi}$ is restricted to $\pi \leq \delta-2$ since, otherwise, the above results become irrelevant. 


\section{Imbeddings}

Here we cite one of the earliest results relating the connectivity of a graph to a topological property of that graph. Other more recent results of this kind can be found in [PluZh98, PluZh02].

\section{DEFINITION}

D22: A graph $G$ is said to be imbeddable in a given surface $S$ if $G$ can be drawn on $S$ without edge crossings.

\section{FACT}

F66: [Co73] Let $G$ be any graph embeddable in a oriented surface of genus $g>0$ (where the genus is, informally, the number of handles on its surface [see Chapter 7 of this Handbook]). Then, $\kappa \leq\lfloor(5+\sqrt{1+48 g}) / 2\rfloor$.

\section{Adjacency Spectrum}

Given a (di)graph $G$ with some associated matrix $A$, a natural problem is to study how much can be said about the structure of $G$ from the spectrum of $A$. This is a major topic in algebraic graph theory, and has been the object of research (see $\S 6.5$ of the Handbook or the classic textbooks D. Cvetković, M. Dragoš, and H. Sach [CvDoSa95], Biggs [Bi94]).

\section{DEFINITIONS}

D23: Given a graph $G$ on $n$ vertices, its adjacency matrix $A=\left(a_{u v}\right)$ is the $n \times n$ matrix indexed by the vertices of $G$ with entries $a_{u v}=1$ if $u$ and $v$ are adjacent and $a_{u v}=0$ otherwise.

D24: The toughness $t$ of a graph $G$ is defined as $t=\min _{S}\{|S| / c(G-S)\}$, where $S$ runs over all vertex-cuts of $G$ and $c(G-S)$ denotes the number of components of $G-S$.

\section{FACTS}

F67: [A195, Br95] Let $G$ be a connected, non-complete $d$-regular graph and let $\lambda$ be the maximum of the absolute values of the eigenvalues of $G$ distinct from $d$. Then, $t>d / \lambda-2$.

Notation: Given a graph $G$, let $D_{2}$ denote the maximum distance between vertex subsets of $G$ with two vertices. (This parameter is a particular case of the so-called conditional diameter, introduced in [BaCaFaFi96].)

F68: [FiGaYe97] Let $G$ be a $d$-regular graph with $D_{2}>1$ and distinct eigenvalues (of its adjacency matrix $A) \lambda_{0}(=d)>\lambda_{1}>\cdots>\lambda_{r}$. Let $P(x):=2\left(x-\lambda_{r}\right) /\left(\lambda_{1}-\lambda_{r}\right)-1$. Then, $\kappa(G) \geq \min \left\{d,\left\lceil\frac{2\left(P(d)^{2}-1\right)(n-2)}{2\left(P(d)^{2}-1\right)+n}\right\rceil\right\}$. 


\section{REMARKS}

R34: Besides Fact F67, Brouwer [Br96] gave some other interesting examples of results about the connectivity of a graph $G$ in terms of its spectrum.

R35: For other results concerning the toughness of a graph, mainly used in the study of vulnerability of network topologies [BoHaKa81], see, for instance, [ChLi02].

R36: Notice that, from Fact F16(b), if $D_{2}=1$ then $G$ is maximally connected. Otherwise, Fact F68 applies.

\section{Laplacian Spectrum}

\section{DEFINITION}

D25: Given a graph $G$, its Laplacian matrix $L$ is defined as $L=D-A$, where $D$ is the diagonal matrix of the vertex degrees and $A$ is the adjacency matrix of $G$ (see, for instance [Bi94]). The Laplacian eigenvalues of $G$ are the eigenvalues of its Laplacian matrix.

TERMinology: The second smallest Laplacian eigenvalue, $\theta_{1}$, usually denoted by $a=$ $a(G)$, is called the algebraic connectivity of $G$ because it has some properties which are similar to those satisfied by the connectivity $\kappa$.

\section{FACTS}

F69: $\quad$ Since the Laplacian matrix $L$ is positive semidefinite, its eigenvalues are all nonnegative, with the first one equal to zero. If $G$ is $d$-regular with (distinct) eigenvalues $\lambda_{0}(=d)>\lambda_{1}>\cdots \geq \lambda_{r}$, then its Laplacian eigenvalues are $\theta_{0}, \theta_{1}, \ldots, \theta_{r}$, where $\theta_{i}=d-\lambda_{i}, i=1,2, \ldots, r$.

F70: [Fi73] Let $G$ be a graph with second smallest Laplacian eigenvalue $a$.

(a) $\kappa \geq a \geq 0$, and $a=0$ if and only if $G$ is not connected.

(b) For any spanning subgraph $H$ of $G$ we have $a(H) \leq a(G)$.

(c) For any vertex subset $U$ of $G$ we have $a(G-U) \geq a(G)-|U|$.

F71: Let $G$ be a $d$-regular graph with $n$ vertices, $D_{2}>1$, and Laplacian eigenvalues $\theta_{0}(=0)<\theta_{1}<\theta_{2}<\cdots<\theta_{r}$. If $d<\frac{n\left(\theta_{r}-\theta_{1}\right)^{2}+8 \theta_{1} \theta_{r}(n-1)}{n\left(\theta_{r}-\theta_{1}\right)^{2}+8 \theta_{1} \theta_{r}}$, then $\kappa=d$.

\section{REMARK}

R37: Fact F71 is just a consequence of Fact F68 in terms of the Laplacian eigenvalues. 


\subsubsection{Symmetry and Regularity}

\section{Boundaries, Fragments, and Atoms}

The concepts of fragment and atom are very useful in the study of connectivity, both in the undirected and the directed case, and, in particular, for (di)graphs with strong symmetries. For graphs, the concept of an atom was introduced independently by Mader [Ma70] and Watkins [Wa70]. The notion of an atom for digraphs was introduced by Chaty [Ch76] and first used extensively by Hamidoune [Ha77, Ha80, Ha81].

Because of the close relationship between a graph $G$ and its corresponding symmetric digraph $G^{*}$, we only give the definitions for digraphs. (For undirected graphs, the corresponding definitions are unsigned.)

\section{DEFINITIONS}

D26: The positive boundary of a vertex subset $F$ in a digraph $G$, denoted $\partial^{+} F$, is the set of vertices that are adjacent from $F$, and the negative boundary, $\partial^{-} F$, is the set of vertices adjacent to $F$.

D27: The positive edge-boundary and the negative edge-boundary, denoted $\omega^{+} F$ and $\omega^{-} F$, respectively, are given by

$$
\begin{aligned}
& \omega^{+} F=\{(u, v) \in E: u \in F \text { and } v \in V-F\} \\
& \omega^{-} F=\{(u, v) \in E: u \in V-F \text { and } v \in F\} .
\end{aligned}
$$

D28: Let $G$ be a strongly connected digraph with connectivity $\kappa$. A vertex subset $F$ is a positive fragment of $G$ if $\left|\partial^{+} F\right|=\kappa$ and $V-\left(F \cup \partial^{+} F\right) \neq \emptyset$, and $F$ is a negative fragment if $\left|\partial^{-} F\right|=\kappa$ and $V-\left(\partial^{-} F \cup F\right) \neq \emptyset$.

D29: Let $G$ be a digraph with edge-connectivity $\lambda$. A vertex subset $F$ is a positive $\alpha$-fragment of $G$ if $\left|\omega^{+} F\right|=\lambda$, and $F$ is a negative $\alpha$-fragment if $\left|\omega^{-} F\right|=\lambda$.

D30: A vertex $u$ of a positive [negative] $\alpha$-fragment $F$ is called interior if none of the edges adjacent from [to] $u$ belongs to $\omega^{+} F\left[\omega^{-} F\right]$.

D31: An atom is a (positive or negative) fragment of minimum cardinality.

\section{EXAMPLE}

E7: For the digraph of Figure 4.7.6, $\kappa=2$ and $F$ is a positive (respectively, negative) fragment with positive (respectively, negative) boundary $\{u, v\}$ (respectively, $\{z, t\}$ ). Analogously, $\omega^{+} F=\{(x, v),(y, u)\}$ and $\omega^{-} F=\{(z, x),(t, y)\}$. In this digraph, each single vertex is an atom.

\section{FACT}

F72: If $F \cup \partial^{+} F \neq V\left[F \cup \partial^{-} F \neq V\right]$, then $\partial^{+} F\left[\partial^{-} F\right]$ is a vertex-cut of $G$. Similarly, if $F$ is a proper (nonempty) subset of $V$, then $\omega^{+} F\left[\omega^{-} F\right]$ is an edge-cut. Using these concepts, we have the following alternative definitions of the connectivity parameters:

$$
\begin{aligned}
& \kappa=\min \left\{\left|\partial^{+} F\right|: F \subset V, F \cup \partial^{+} F \neq V \text { or }|F|=1\right\} \\
& \lambda=\min \left\{\left|\omega^{+} F\right|: F \text { is a nonempty, proper subset of } V\right\}
\end{aligned}
$$




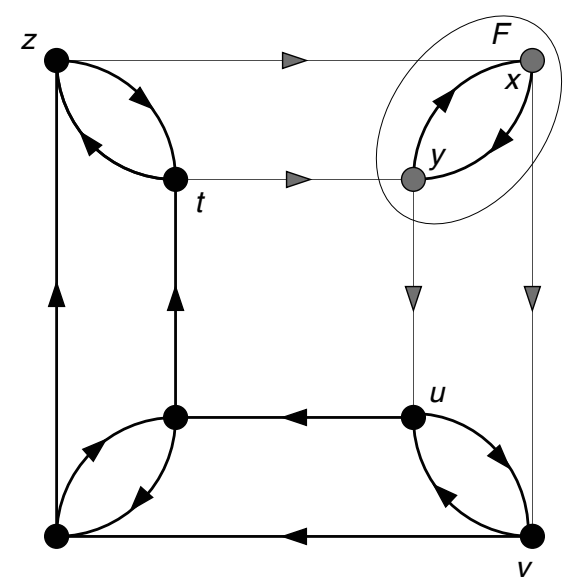

Figure 4.7.6: $F$ is a fragment.

\section{Fragments and Atoms in Undirected Graphs}

\section{FACTS}

F73: [Wa70, Ma71a] In a connected graph, any two distinct atoms are disjoint.

F74: [Ma71a] Let $G$ be a graph with order $n$ and connectivity $\kappa$. Let $F_{1}$ and $F_{2}$ be distinct minimal fragments of $G$, with at most $n-3 \kappa / 2$ vertices. Then $F_{1} \cap F_{2}=\emptyset$.

F75: [Ma71a] Let $G$ be a graph with connectivity $\kappa$. If $T$ is a disconnecting set with $\kappa$ vertices and $A$ is an atom, then either $A \subset T$ or $A \cap T=\emptyset$.

\section{REMARKS}

R38: To quote a personal communication from Watkins [Wa02]: "It is an amazing coincidence that Prof. Mader and I not only conceived of the notion of 'atom' independently and simultaneously, but we also accorded this notion almost identical names." In fact, Watkins [Wa70] used the term "atomic part," while Mader [Ma70] used the term "kleinstes Glie" (that is, "smallest member"). Then, in a subsequent paper, Mader [Ma71a] mentioned the main result in [Wa70] on atomic parts, and deduced it from his results. Moreover, inspired by Watkins' terminology, he first used the simpler name "atom."

R39: Fact F73 was proved by Watkins for transitive graphs, whereas the general case is due to Mader.

R40: As already mentioned, the seminal papers on atoms are those of Mader [Ma70] and Watkins [Wa70]. Notice that Fact F75 is a generalization of Fact F73, and it is considered as the more important property of an atom.

R41: Results on atoms and the connectivity of infinite graphs can be found in [JuWa77] and $[\mathrm{Ha} 89]$. 


\section{Fragments and Atoms in Digraphs}

The results above can be seen as consequences of the corresponding directed versions, which are due to Hamidoune.

\section{FACTS}

F76: [Ha77] Let $G$ be a connected digraph with a positive (negative) atom $A$ and a positive (negative) fragment $F$. Then, either $A \subset F$ or $A \cap F=\emptyset$. In particular, two distinct positive (negative) atoms are disjoint.

F77: [Ha80] If $G$ is a connected digraph with $\lambda<\delta^{+}\left(\lambda<\delta^{-}\right)$, then every positive (negative) $\alpha$-fragment contains an interior vertex.

\section{REMARKS}

R42: Contrary to the case of graphs, where the presence of an atom is always assured, a digraph does not necessarily have an atom with a prescribed sign.

R43: Fact F77 implies Jolivet's theorem (Fact F22).

\section{Graphs with Symmetry}

Graphs with high symmetry often have "good" properties, and their study has special relevance to other areas of mathematics. In particular, the results here show that, for connected graphs, high symmetry goes hand in hand with high connectivity. Graph automorphisms and symmetry are discussed in $\S 6.1$ and $\S 6.2$.

\section{DEFINITION}

D32: A (di)graph $G$ is vertex-transitive (or vertex-symmetric) if for any vertices $u, v$ there is an automorphism of $G$ which maps $u$ into $v$. Similarly, $G$ is called edgetransitive (or edge-symmetric) if for any (possibly oriented) edges $u v, w z$ there is an automorphism of $G$ that maps $u v$ into $w z$.

\section{FACTS}

F78: $[$ Ma70, Ma71b] Let $G$ be a vertex-symmetric connected graph with degree $d \geq 3$. Then, $\lambda=d, \kappa \geq 2\lfloor d / 3\rfloor+2$ if $d>3$, and $\kappa=3$ otherwise. Furthermore, if $G$ does not contain $K_{4}$, then $\kappa=d$.

F79: $[$ Ma70] Let $G$ be an edge-symmetric connected graph with degree $d$. Then $\kappa=\lambda=d$.

F80: [Ma70, Wa70] Let $G$ be a vertex-transitive graph with an atom $A$. Then the subgraph $G(A)$ induced on $A$ is also vertex-transitive. Moreover, the set of atoms of $G$ constitutes a partition of $V(G)$.

F81: [Ha77] Let $G$ be a vertex-transitive digraph with a positive (negative) atom $A$. Then, the induced subdigraph $G(A)$ is also vertex-transitive. Furthermore, the set of positive (negative) atoms of $G$ constitutes a partition of $V(G)$. 
F82: [Ha81] Let $G$ be a vertex-symmetric strongly connected digraph with (constant) outdegree $d^{+}$. Then $\lambda=d^{+}$and $\kappa \geq \frac{1}{2} d^{+}$. Moreover, if $G$ is an oriented graph, then $\kappa \geq \frac{2}{3} d^{+}$.

\section{REMARKS}

R44: The inequality in Fact F78, which is best possible, is a consequence of Fact F73 and, for $d \not \equiv 2 \bmod 3$, it is an improvement of a result of Watkins [Wa70], who showed that $\kappa>(2 / 3) d$.

R45: From Facts F80 and F81, the order of a (positive or negative) atom of $G$ divides the order of $G$. Consequently, every connected vertex-transitive (di)graph with a prime number of vertices is maximally connected $(\kappa=\lambda=\delta)$. In fact, it is known that such (di)graphs must be Cayley (di)graphs of cyclic groups.

R46: By Fact F76, Hamidoune [Ha77] proved Kameda's result stating that every minimal $k$-connected digraph has one vertex of out-degree or in-degree $k$ [Ka74], and Hamidoune also proved that every edge-transitive digraph is maximally connected.

\section{Cayley Graphs}

The Cayley graphs are among the most interesting vertex-symmetric (di)graphs, mainly because of their relationship with group theory (see $\S 6.1$ and $\S 6.2$ ). In particular, the study of the connectivity of Cayley graphs has striking connections with some key results in additive number theory, such as the well-known Cauchy-Davenport theorem: If $p$ is a prime number and $A, B$ are two nonempty subsets of the cyclic group $Z_{p}$, then either $A+B=Z_{p}$ or $|A+B| \geq|A|+|B|-1$.

\section{DEFINITIONS}

D33: Let $\Gamma$ be a finite group with identity element $e$ and generating set $S \subset \Gamma-\{e\}$. The Cayley digraph $G=(\Gamma, S)$ has vertices labeled with the elements of $\Gamma$, and edges of the form $(u, u g)$ where $g \in S$. In particular, when $S^{-1}=S$ (where $S^{-1}=\left\{x^{-1}: x \in\right.$ $S\}$ ) we obtain a symmetric Cayley digraph or, simply, a Cayley graph.

D34: If $\Gamma$ is a cyclic group, then the Cayley graph is called a circulant graph.

D35: A generating set $S$ of a group $\Gamma$ is called minimal when any proper subset $S^{\prime} \subset S$ does not generate $\Gamma$.

D36: The symmetric group on $n$ elements, denoted $\Sigma_{n}$, is the group of all permutations of the set $\{1,2, \ldots, n\}$.

D37: Let $H$ be a subgroup of a group $G$, and let $x \in G$. Then the set $x H=\{x h \mid h \in H\}$ is a left coset of $G$ with respect to $H$.

\section{FACTS}

F83: [Im79] Let $S$ be a generating set of the symmetric group $\Gamma=\Sigma_{n}$ with $n \geq 5$, such that $x S x^{-1}=S$ for every $x \in \Gamma$. Then, the Cayley digraph $(\Gamma, S)$ is maximally connected (that is, $\kappa=|S|$ ). 
F84: [Ha84] Let $\Gamma$ be a finite group with identity $e$ and generating set $S$. Let $A$ be a positive (respectively, negative) atom of $(\Gamma, S)$ containing $e$. Then $A$ is the subgroup of $\Gamma$ generated by $S \cap A$, and the positive (respectively, negative) atoms of $(\Gamma, S)$ are the left cosets of $\Gamma$ with respect to $A$.

F85: [Ha84] Let $\Gamma$ be a finite group with a minimal generating set $S$. Let $S^{\prime} \subset S^{-1}$. Then, the Cayley digraph $\left(\Gamma, S \cup S^{\prime}\right)$ is maximally connected.

F86: [HaSe96] Let $\Gamma$ be an Abelian group of order $n$ and let $S$ be a generating subset of $\Gamma$ such that $|S \cup\{0\}| \leq n-1$. Let $D$ be the diameter of $G=(\Gamma, S)$. Then there is a vertex-cut of size less than $(4 n \ln (n / 2)) / D$ whose deletion separates $G$ into a negative fragment $B$ and a positive fragment $\bar{B}$ such that $|B|=|\bar{B}|$. Moreover, $G$ can be separated into two equal parts of size $|B|$ by deleting less than $(8 e /|S|) n^{1-1 /|S|} \ln (n / 2)$ vertices.

\section{REMARKS}

R47: Fact F84, due to Hamidoune, provides a very short proof of Fact F83.

R48: For the case of Cayley graphs, Fact F85 was previously proved by Godsil [Go81]. Subsequently, Akers and Krishnamurthy [AkKr87], Hamidoune, Lladó, and Serra [HaLlSe92], and Alspach [Als92] improved these results by considering Cayley (di)graphs with a hierarchical generating set (that is, when the group generated by the first $k$ generators is a proper subgroup of the group generated by the first $k+1$ for each $k)$.

\section{Circulant Graphs}

Because of their circular symmetry, circulant graphs have been proposed as good models for local area network topologies, where they are called loop networks. In this context, other good topologies are provided by Cayley graphs of Abelian groups, also called loop networks (see [BeCoHs95], [BoTi84]).

\section{FACTS}

F87: [Ha84] Let $\Gamma$ be the cyclic group $Z_{n}$. Let $S$ be the strictly increasing sequence of $s$ integers $(1=) b_{1}<b_{2}<\cdots<b_{s}(<n)$ such that $b_{i+1}-b_{i} \geq \min \left\{2, b_{i}-b_{i-1}\right\}$ for $i=2,3, \ldots, s-1$. Then the circulant digraph $(\Gamma, S)$ is maximally connected $(\kappa=s)$.

F88: [Ha84] Let $\Gamma$ be the cyclic group $Z_{n}$. Let $S$ be the strictly increasing sequence of $s$ integers $(1=) b_{1}<b_{2}<\cdots<b_{s}(<n / 2)$ such that $b_{i+1}-b_{i} \geq \min \left\{2, b_{i}-b_{i-1}\right\}$ for $i=2,3, \ldots, s-1$, and let $S^{\prime} \subset-S$, where $-S$ denotes the set of (additive) inverses of the elements in $S$. Then the circulant digraph $\left(\Gamma, S \cup S^{\prime}\right)$ is maximally connected $\left(\kappa=\left|S \cup S^{\prime}\right|\right)$.

F89: The Cauchy-Davenport theorem is equivalent to stating that, for any generating set $S \subset Z_{p}, p$ prime, the Cayley digraph $\left(Z_{p}, S\right)$ is maximally connected (that is, $\kappa=|S|)$. 


\section{REMARKS}

R49: The case $S^{\prime}=-S$ in Fact F88 (that is, for circulant graphs) was proved in [BoFe70] using the "convexity conditions" $b_{i+1}-b_{i} \geq b_{i}-b_{i-1}$ (see also [BoTi84]).

R50: Fact F89, noted by Hamidoune, is a bridge between additive number theory and graph theory. (For a comprehensive survey on the subject, we refer the reader to [Ha96].)

\section{Distance-Regular Graphs}

The concept of distance-regularity was introduced by Biggs [Bi71] in the early 1970s, by changing a symmetry-type requirement, that of distance-transitivity, to a regularitytype condition concerning the cardinality of some vertex subsets. Distance-regular graphs have important connections with other branches of mathematics, such as geometry, coding theory, and group theory, as well as with other areas of graph theory. In our context, their high regularity seems also to induce a high degree of connectedness.

\section{DEFINITIONS}

D38: Let $G$ be a regular graph with diameter $D$ and let $k$ be an integer between 1 and $D$. Graph $G$ is said to be distance-regular if, for any two vertices $u$ and $v$ with $\operatorname{dist}(u, v)=k$, the numbers $c_{k}, a_{k}$, and $b_{k}$ of vertices that are adjacent to $v$ and whose distance from $u$ is $k-1, k$, and $k+1$, respectively, depend only on $k$.

D39: An $n$-vertex $k$-regular graph $G$ is called $(n, k ; a, c)$-strongly-regular if any two adjacent vertices have $a$ common neighbors and any two non-adjacent vertices have $c$ common neighbors.

\section{FACTS}

F90: Let $G$ be a connected graph. Then $G$ is strongly-regular if and only if $G$ is distance-regular of diameter two.

F91: Every strongly regular graph is maximally edge-connected.

F92: [BrMe85] Every strongly regular graph is maximally connected and super- $\kappa$.

F93: [BrKo09] Every distance-regular graph is maximally connected. If the degree is at least 3 , it is also super- $\kappa$.

\section{REMARKS}

R51: Fact F91 is a consequence of Facts F3 and F90.

R52: Fact F93 was a long standing conjecture of Brouwer [Br96]. The result was previously proved for some families of distance-regular graphs, such as the so-called odd graphs $O_{k}$ (having the $k$-subsets of a $(2 k-1)$-set as its vertices and adjacencies defined by void intersection); see [Gh92].

R53: Fact F93 implies a previous conjecture of Godsil [Go81], stated in the context of association schemes, that every distance-regular graph is maximally edge-connected. In fact, this last result was proved by Brouwer and Haemers in [BrHa05]. 


\subsubsection{Generalizations of Connectivity Parameters}

The standard connectivity parameters have been generalized in different ways, giving rise to numerous articles; see, for instance, [BaBeLiPi87], [BeOePi02], [Ha83], [Wo73]. Here we will consider several examples, some of which have special relevance to the study of network vulnerability.

\section{Conditional Connectivity}

The next two definitions generalize the concept of superconnectivity.

\section{DEFINITIONS}

D40: Given a graph $G=(V, E)$ and a nonnegative integer $s$, a vertex subset $V^{\prime} \subset V$ is said to be $n$-trivial if it contains the boundary $\partial(H)$ of some subgraph $H \subset G$ with $s^{\prime}$ vertices, $1 \leq s^{\prime} \leq s$. Similarly, an edge subset $E^{\prime} \subset E$ is said to be $s$-trivial if it contains the edge-boundary $\omega(H)$ of some subgraph $H \subset G$ with $s^{\prime}$ vertices, $1 \leq s^{\prime} \leq s$.

D41: The conditional connectivity $\kappa_{s}$ of a graph $G$ is the minimum cardinality of a disconnecting set that is not $s$-trivial. The conditional edge-connectivity $\lambda_{s}$ of $G$ is the minimum cardinality of a disconnecting edge set that is not $s$-trivial.

\section{FACT}

F94: [FaFi89, FiFaEs90, FaFi94] Let $G$ be a graph with minimum degree $\delta>1$, diameter $D$ and girth $g$. Let $\ell=\left\lfloor\frac{g-1}{2}\right\rfloor$.

(a) If $D \leq 2 \ell$, then $\lambda_{0}=\delta$.

(b) If $D \leq 2 \ell-1$, then $\kappa_{0}=\delta$ and $\lambda_{1} \geq 2 \delta-2$.

(c) If $D \leq 2 \ell-2$, then $\kappa_{1} \geq 2 \delta-2$ and $\lambda_{2} \geq 3 \delta-4$.

(d) If $D \leq 2 \ell-3$, then $\kappa_{2} \geq 3 \delta-4$.

\section{CONJECTURE}

C1: $[\mathrm{FaFi94}]$

(a) If $D \leq 2 \ell-s$, then $\lambda_{s} \geq(s+1) \delta-2 s$.

(b) If $D \leq 2 \ell-s-1$, then $\kappa_{s} \geq(s+1) \delta-2 s$.

\section{REMARKS}

R54: Harary [Ha83] introduced the general concept of conditional connectivity. In our context, the graphs are assumed to be those for which $\kappa_{s}$ and $\lambda_{s}$ are well-defined.

R55: Note that the conditional connectivities $\kappa_{0}$ and $\lambda_{0}$ correspond to the standard connectivities $\kappa$ and $\lambda$ (thus, Fact F94 generalizes Fact F37). If $\kappa_{1}>\delta$, then $G$ is super- $\kappa$, and if $\lambda_{1}>\delta$, then $G$ is super- $\lambda$.

R56: The conjecture above was proved to be true for even $s$ (provided that $\delta>$ 2 and $\ell>(s+1) / 2$ ) [FaFi96b]. Moreover, if $s$ is large enough in comparison with the minimum degree $\delta$, further improvements of the sufficient conditions were given in [BaCaFaFi97b, Ba99]. 


\section{Restricted Connectivity}

D42: The restricted edge-connectivity $\lambda^{\prime}=\lambda^{\prime}(G)$, introduced by Esfahanian and Hakimi [Es88], is the minimum cardinality over all restricted edge-cuts, i.e., those edge-cuts $S$ such that there are no isolated vertices in $G-S$.

D43: The minimum edge-degree of $G$ is $\xi=\xi(G)=\min \{\operatorname{deg}(u)+\operatorname{deg}(v)-2: u v \in$ $E\}$.

D44: A graph is said to be $\lambda^{\prime}$-optimal if $\lambda^{\prime}=\xi$.

D45: Given a graph $G=(V, E)$, an edge set $S \subset E$ is called a $k$-restricted-edge-cut if $G-S$ is disconnected and every component of $G-S$ has at least $k$ vertices.

D46: The $k$-restricted-edge-connectivity of $G$, denoted by $\lambda^{(k)}=\lambda^{(k)}(G)$, is defined as the cardinality of a minimum $k$-restricted-edge-cut.

D47: The edge-boundary $\omega(F)$ is called a $\lambda^{(k)}$-cut if $|\omega(F)|=\lambda^{(k)}$, and $F \subset V$ is called a $k$-fragment of $G$. A minimum $k$-fragment is called a $k$-atom, and its cardinality is denoted $a_{k}(G)=a_{k}$.

D48: The minimum $k$-edge-degree of $G$ is $\xi_{k}=\xi_{k}(G)=\min \{|\omega(U)|: \emptyset \neq U \subset$ $V(G),|U|=k$ and $G[U]$ is connected $\}$.

D49: A graph with $k$-restricted edge cuts is said to be $\lambda^{(k)}$-optimal if $\lambda^{(k)}=\xi_{k}$.

D50: A graph $G$ is super $k$-restricted edge connected, or super- $\lambda^{(k)}$, if $G$ is $\lambda^{(k)}$ optimal and the deletion of every $\lambda^{(k)}$-cut isolates a component with $k$ vertices; that is, if every $k$-fragment $X$ has cardinality $|X| \in\{k, n-k\}$.

\section{REMARKS}

R57: Esfahanian and Hakimi [Es88] showed that each connected graph of order $n \geq 4$ except a star has restricted edge-cuts and satisfies $\lambda^{\prime} \leq \xi$.

R58: The restricted edge-connectivity $\lambda^{\prime}$ corresponds to the 2-restricted-edge-connectivity $\lambda^{(2)}$ and also to the conditional connectivity $\lambda_{1}$ defined in Definition D41.

R59: Furthermore, the $k$-restricted-edge-connectivity $\lambda^{(k)}$ corresponds to the conditional connectivity $\lambda_{k-1}$ given in Definition D41, for any $k \geq 1$.

R60: If $G$ is super- $\lambda^{(k)}$, then $a_{k}=k$.

\section{FACTS}

F95: $\quad$ BaGVMa06] Every graph with girth $g$ and $\delta \geq 2$ is super- $\lambda$ and has $\lambda^{\prime}=\xi$ if its diameter $D \leq g-2$.

F96: [BaGVMa06] Every graph $G$ with girth $g$ odd and $\delta \geq 2$ is super- $\lambda$ and has $\lambda^{\prime}=\xi$ if $D=g-1$ and either of the following assertions holds.

(i) All pairs $u, v$ of vertices at distance $d(u, v)=g-1$ are such that neither vertex $u$ nor $v$ lies on a cycle of length $g$. 
(ii) $\left|N_{(g-1) / 2}(u) \cap N_{(g-1) / 2}(v)\right| \geq 3$ for all pairs $u, v$ of vertices at distance $d(u, v)=$ $g-1$ where $N_{h}(u)=\{w \in V(G): d(u, w)=h\}$.

F97: [BaCeDiGVMa05] Every graph with girth $g$ even and $\delta \geq 2$ is super- $\lambda$ and has $\lambda^{\prime}=\xi$ if $D=g-1$ and only $\delta-1$ vertices are mutually at distance $g-1$ apart.

F98: [BaLiMi08] Every graph $G$ with girth $g$, minimum degree $\delta \geq 3$, and diameter $D$ is super- $\lambda^{\prime}$ if $D \leq g-3$ or if the diameter of the line graph satisfies $D(L(G)) \leq g-3$.

F99: [ZhYu05] Let $G$ be a connected graph with order at least $2(\delta+1)$ not isomorphic to any $G_{s, \delta}^{*}$, where $G_{s, \delta}^{*}$ is the graph obtained from $s$ copies of $K_{\delta}$ by adding a new vertex $u$ and joining $u$ to every other vertex. Then for any $k \leq \delta+1, G$ has $k$-restricted edge cuts and $\lambda^{(k)} \leq \xi_{k}$.

F100: [ZhYu07] Let $G$ a connected graph on $n \geq 2 k$ vertices. If $\operatorname{deg}(u)+\operatorname{deg}(v) \geq$ $n+2 k-3$, for every pair of nonadjacent vertices $u$ and $v$, then $G$ is $\lambda^{(k)}$-optimal.

F101: [BoUeVo02, XuXu02] For $k=2,3$, a graph with $\lambda^{(k)}$-cuts has $\lambda^{(k)}=\xi_{k}$ if and only if $a_{k}=k$.

F102: [BaGMMa09] Let $G$ be a graph with $\lambda^{(k)}$-cuts and such that $\lambda^{(k)} \leq \xi_{k}$. Then $G$ is $\lambda^{(k)}$-optimal if $a_{k}=k$. Moreover, $a_{k}=k$ follows if $G$ is $\lambda^{(k)}$-optimal and either of the following conditions holds.

(i) $\delta \geq 2 k-1$.

(ii) $\delta \geq k+1$ and $g \geq k+1$.

F103: [BaGMMa09] Let $G$ be a graph with $\lambda^{(k)}$-cuts such that $\lambda^{(k)} \leq \xi_{k}$ and $\lambda^{(k+1)}$ exists. Then $G$ is super- $\lambda^{(k)}$ if and only if $\lambda^{(k+1)}>\xi_{k}$.

F104: [ZhLi10] Let $G$ be a graph with $\lambda^{(k)}$-cuts, $k \geq 3$, girth $g \geq 5$, minimum degree $\delta \geq k$, and diameter $D$. Then $G$ is $\lambda^{(k)}$-optimal if either of the following conditions holds.

(i) $D \leq g-4$ when $g$ is even, or $D \leq g-3$ when $g$ is odd;

(ii) $D \leq g-3$ and $\delta \geq 2 k-3$.

F105: $\quad\left[\right.$ BaGV10] Let $G$ be a graph with $\lambda^{(k)}$-cuts, girth $g$, minimum degree $\delta \geq$ $\max \{3, k\}$, and diameter $D$. Then $G$ is super- $\lambda^{(k)}$ if either of the following conditions holds.

(i) $D \leq g-4$ when $g$ is even, or $D \leq g-4$ when $g$ is odd and $\delta \geq k+1$;

(ii) the diameter of the line graph is $D(L(G)) \leq g-4$. 


\section{Distance Connectivity}

Here we consider a generalization of the concepts of connectivity and edge-connectivity of a (di)graph, introduced in [FiFa94] and [BaCaFi96], which takes into account the distance between vertices.

\section{DEFINITIONS}

D51: Let $G=(V, E)$ be a digraph. Given $u, v \in V$ such that $(u, v) \notin E$, recall (from $\S 4.1)$ that a set $S \subset V-\{u, v\}$ is called a $(u \mid v)$-set if there is no $u-v$ path in $G-S$, and $\kappa(u \mid v)$ is the minimum cardinality of a $(u \mid v)$-set. Similarly, a given edge-set $T \subset E$ is called a $(u \mid v)$-edge-set for some $u, v \in V$ if there is no $u$-v path in $G-T$, and $\lambda(u \mid v)$ is the minimum cardinality of a $(u \mid v)$-edge-set.

D52: Let $G=(V, E)$ be a digraph with diameter $D$. Given $t, 1 \leq t \leq D$, the $t$-distance connectivity of $G$, denoted by $\kappa(t ; G)=\kappa(t)$, is defined as $\kappa(t)=\min \{\kappa(u \mid v): u, v \in$ $V$, $\operatorname{dist}(u, v) \geq t\}$ if $t \geq 2$, and $\kappa(1)=\kappa$, where $\kappa$ is the standard connectivity of $G$. Analogously, the $t$-distance edge-connectivity is $\lambda(t ; G)=\lambda(t)=\min \{\lambda(u \mid v): u, v \in$ $V, \operatorname{dist}(u, v) \geq t\}$ for $t \geq 1$.

\section{FACTS}

F106:

(a) $\kappa=\kappa(1)=\kappa(2) \leq \kappa(3) \leq \cdots \leq \kappa(D)$.

(b) $\lambda=\lambda(1) \leq \lambda(2) \leq \cdots \leq \lambda(D)$.

F107: Let $G$ be a digraph with minimum degree $\delta>1$ and semigirth $\ell$ (see Definition D12).

(a) If $\lambda<\delta$ then $D \geq 2 \ell+1$ and $\lambda=\lambda(2 \ell+1)$.

(b) If $\kappa<\delta$ then $D \geq 2 \ell$ and $\kappa=\kappa(2 \ell)$.

\section{F108:}

(a) $\lambda=\delta$ if and only if $D \leq 2 \ell$ or $\lambda(2 \ell+1) \geq \delta$.

(b) $\kappa=\delta$ if and only if $D \leq 2 \ell-1$ or $\kappa(2 \ell) \geq \delta$.

F109: Every digraph with distance connectivity $\lambda(3) \geq \delta$ has maximum edge-connectivity.

F110: Let $G$ be an undirected graph with associated symmetric digraph $G^{*}$. Since a minimum $t$-distance disconnecting set of $G^{*}$ cannot contain digons, $\kappa\left(t ; G^{*}\right)=\kappa(t ; G)$ and $\lambda\left(t ; G^{*}\right)=\lambda(t ; G)$.

F111: Let $G$ be an undirected graph with girth $g$ and $\delta>1$.
(a) If $\lambda<\delta$ then $\begin{cases}D \geq g \text { and } \lambda=\lambda(g), & \text { g odd, } \\ D \geq g-1 \text { and } \lambda=\lambda(g-1), & \text { g even. }\end{cases}$
(b) If $\kappa<\delta$ then $\begin{cases}D \geq g-1 \text { and } \kappa=\kappa(g-1), & \text { g odd, } \\ D \geq g-2 \text { and } \kappa=\kappa(g-2), & \text { g even. }\end{cases}$ 
F112:

(a) $\lambda=\delta$ if and only if $\begin{cases}D \geq g-1 \text { or } \lambda(g) \geq \delta, & \text { g odd, } \\ D \geq g-2 \text { or } \lambda(g-1) \geq \delta, & \text { g even }\end{cases}$

(b) $\kappa=\delta$ if and only if $\begin{cases}D \geq g-2 \text { or } \kappa(g-1) \geq \delta, & \text { g odd, } \\ D \geq g-3 \text { or } \kappa(g-2) \geq \delta, & \text { g even. }\end{cases}$

F113: Any graph with distance connectivity $\lambda(3) \geq \delta$ has maximum edge-connectivity.

\section{REMARKS}

R61: In Fact F108, since $\kappa(t)$ and $\lambda(t)$ are defined only for $t \leq D$, the two sufficient conditions on the diameter and the distance connectivity are complementary to one another.

R62: Since the semigirth of any digraph is at least one, Fact F107(a) implies Fact F109, which complements Jolivet's result (Fact F22).

R63: Fact F111 follows from Fact F107 by considering Fact F110 and $\ell\left(G^{*}\right)=\lfloor(g-$ $1) / 2\rfloor$.

\section{High Distance Connectivity}

\section{DEFINITIONS}

D53: Given a vertex $u$ of a digraph $G$, the out-and in-eccentricity of $u$ are ecc $^{+}(u)=$ $\max _{v \in V}\{\operatorname{dist}(u, v)\}$ and $e c c^{-}(u)=\max _{v \in V}\{\operatorname{dist}(v, u)\}$, respectively.

D54: For any integer $t, 1 \leq t \leq D$, the minimum $t$-degree of a digraph $G$ is $\delta(t)=\min \left\{\delta^{+}(t), \delta^{-}(t)\right\}$, where $\delta^{+}(t)=\min _{u \in V}\left\{d e g^{+}(u): e c c^{+}(u) \geq t\right\}$ and $\delta^{-}(t)=$ $\min _{u \in V}\left\{\operatorname{deg}^{-}(u): \operatorname{ecc}^{-}(u) \geq t\right\}$.

D55: A connected digraph $G$ with diameter $D$ is said to be $s$-geodetic, for some $1 \leq s \leq D$, if any two vertices of $G$ are joined by at most one path of length less than or equal to $s$. If $s=D$, the digraph is called strongly geodetic (see [BoKoZn68], $[\mathrm{PlZn74}])$.

\section{FACTS}

F114: $\delta=\delta(1)=\cdots=\delta(r) \leq \delta(r+1) \leq \cdots \leq \delta(D)$.

F115: For any $t, 1 \leq t \leq D, \kappa(t) \leq \lambda(t) \leq \delta(t)$.

TERMINOLOGY: A digraph $G$ is called maximally $t$-distance connected when $\kappa(t)=$ $\lambda(t)=\delta(t)$, and maximally $t$-distance edge connected when $\lambda(t)=\delta(t)$.

F116: If a digraph $G$ is maximally connected, then $G$ is maximally $t$-distance connected for any $1 \leq t \leq r$. 
F117: [BaCaFaFi97a] Let $G$ be an $s$-geodetic digraph. Then,

(a) $\lambda(t)=\min \{\delta(t), \lambda(2 \ell+1)\}$, for any $t \leq 2 s+1$.

(b) $\kappa(t)=\min \{\delta(t), \kappa(2 \ell)\}$, for any $t \leq 2 s$.

F118: [BaCaFaFi97a] Let $G$ be an $s$-geodetic digraph.

(a) $G$ is maximally $t$-distance connected for any $t \leq 2 s$ if $D \leq 2 \ell-1$.

(b) $G$ is maximally $t$-distance edge connected for any $t \leq 2 s+1$ if $D \leq 2 \ell$.

F119: Let $G$ be a graph with girth $g$ and diameter $D$. Then, for any $1 \leq t \leq D$,

(a) $G$ is maximally $t$-distance edge connected if $\begin{cases}D \leq g-1, & \text { g odd, } \\ D \leq g-2, & \text { g even. }\end{cases}$

(b) $G$ is maximally $t$-distance connected if $\begin{cases}D \leq g-2, & \text { g odd, } \\ D \leq g-3, & \text { g even. }\end{cases}$

\section{Maximal Connectivity}

Instead of looking for minimum disconnecting sets, we can consider those (minimal) disconnecting sets with maximum cardinalities. This leads to considering the following connectivity parameters.

NOTATION: Denote by $\kappa_{\max }$ and $\lambda_{\max }$ the maximum cardinality of a minimal disconnecting (vertex) set and a minimal disconnecting edge set, respectively.

\section{FACTS}

F120: $\kappa_{\max } \geq \kappa$ and $\lambda_{\max } \geq \lambda$.

F121: [PeLaHe86] For any non-trivial graph $G$ with order $n$ and maximum degree $\Delta \neq n-1$ we have $\kappa_{\max } \leq \lambda_{\max }$. Furthermore, if $\mathrm{G}$ is 2 -connected, then $\lambda_{\max } \geq \Delta$.

F122: $[$ PeLaHe86] Let $G$ be an $n$-vertex graph with minimum degree $\delta$.

(a) If $\delta \geq\lfloor n / 2\rfloor$, then $\lambda_{\max } \geq \delta$.

(b) If $\delta \geq\lfloor(n+i) / 2\rfloor$ for some $i$ with $1 \leq i \leq n / 2$, then $\lambda_{\max } \geq i\lfloor(n-i+2) / 2\rfloor$.

(c) If $\delta \geq\lfloor(n+i) / 2\rfloor$ for some $i$ with $n / 2<i<n-2$, then $\lambda_{\max } \geq\lceil n / 2\rceil \cdot\lfloor(i+1) / 2\rfloor$. 


\section{Hamiltonian Connectivity}

\section{DEFINITIONS}

D56: A graph $G$ is hamiltonian connected if between any pair of vertices $u, v$ there is a hamiltonian $u-v$ path in $G$.

D57: A graph $G k$-leaf-connected if $|V(G)|>k$ and for each subset $S$ of $V(G)$ with $k=|S|$ there exists a spanning tree $T$ with precisely $S$ as the set of endvertices (vertices of degree 1).

\section{FACTS}

F123: [GuWa86] Let $u$ and $v$ be non-adjacent vertices of $G$ with $d(u)+d(v) \geq$ $|V(G)|+k-1$. If $G+u v$ is $k$-leaf-connected, then $G$ is $k$-leaf-connected.

F124: [GuWa86] For all natural numbers $n, k, 2 \leq k<n-2$, there are $k$-leaf-connected graphs with $\lceil(k+1) n / 2\rceil$ edges (the minimum number of edges that a $k$-leaf-connected graph on $n$ vertices can have).

\section{REMARK}

R64: The generalization of the concept of hamiltonian connectivity (Definition D57) is due to Murty. Notice that $G$ is hamiltonian-connected if and only if $G$ is 2-leafconnected.

\section{References}

[Ai67] M. Aigner, On the linegraph of a directed graph. Math. Z. 102 (1967), 56-61.

[AkKr87] S.B. Akers and B. Krishnamurthy, On group graphs and their fault tolerance. IEEE Trans. Comput. 36 (1987), 885-888.

[A195] N. Alon, Tough Ramsey graphs without short cycles. J. Algebraic Combin. 4 (1995), 189-195.

[Als92] B. Alspach, Cayley graphs with optimal fault tolerance. IEEE Trans. Comput 41 (1992), 1337-1339.

[ArGoMoSe07] G. Araujo, D. González Moreno, J.J. Montellano, and O. Serra, On upper bounds and connectivity of cages. Australas. J. Combin. 38 (2007), 221228.

[AyFr70] J.N. Ayoub and I.T. Frisch, On the smallest-branch cuts in directed graphs. IEEE Trans. Circuit Theory CT-17 (1970), 249-250.

[Ba99] M.C. Balbuena, Extraconnectivity of $s$-geodetic digraphs and graphs. Discrete Math. 195 (1999), 39-52.

[BaBeLiPi87] K.S. Bagga, L.W. Beineke, M.J. Lipman, and R.E. Pippert, On the edgeintegrity of graphs. Congressus Numerantium 60 (1987), 141-144. 
[BaCaFaFi96] M.C. Balbuena, A. Carmona, J. Fàbrega, and M.A. Fiol, On the connectivity and the conditional diameter of graphs and digraphs. Networks 28 (1996), 97-105.

[BaCaFaFi97a] M.C. Balbuena, A. Carmona, J. Fàbrega, and M.A. Fiol, On the order and size of $s$-geodetic digraphs with given connectivity. Discrete Math. 174 (1997), $19-27$.

[BaCaFaFi97b] M.C. Balbuena, A. Carmona, J. Fàbrega, and M.A. Fiol, Extraconnectivity of graphs with large minimum degree and girth. Discrete Math. 167/168 (1997), 85-100.

[BaCaFi96] M.C. Balbuena, A. Carmona, and M.A. Fiol, Distance connectivity in graphs and digraphs. J. Graph Theory 22 (1996), 281-292.

[BaCeDiGVMa05] C. Balbuena, M. Cera, A. Diánez, P. García-Vázquez, and X. Marcote, Sufficient conditions for $\lambda^{\prime}$-optimality of graphs with small conditional diameter. Inf. Process. Letters 95 (2005), 429-434.

[BaCeDiGVMa06] C. Balbuena, M. Cera, A. Diánez, P. García-Vázquez, and X. Marcote, On the restricted connectivity and superconnectivity in graphs with given girth. Discrete Math. 307 (2007), 659-667.

[BaCeDiGVMa07] C. Balbuena, M. Cera, A. Diánez, P. García-Vázquez, and X. Marcote, Connectivity of graphs with given girth pair. Discrete Math. 307 (2007), $155-162$.

[BaGVMa06] C. Balbuena, P. García-Vázquez, and X. Marcote, Sufficient conditions for $\lambda^{\prime}$-optimality in graphs with girth $g$. J. Graph Theory 52 (2006), 73-86.

[BaGV10] C. Balbuena and P. García-Vázquez, Edge fault tolerance analysis of super k-restricted connected networks. Appl. Math. Comput. 216 (2010), 506-513.

[BaGVMo11] C. Balbuena, P. García-Vázquez, and L. P. Montejano, Superconnectivity of graphs with odd girth $g$ and even girth $h$. Discrete Appl. Math. 159 (2011), 9199 .

[BaGMMa09] C. Balbuena, D. González-Moreno, and X. Marcote, On the 3-restricted edge connectivity of Permutation Graphs. Discrete Appl. Math. 157 (2009), 15861591.

[BaLiMi08] C. Balbuena, Y. Lin, and M. Miller, Diameter-sufficient conditions for a graph to be super restricted connected. Discrete Applied Math 156 (2008), 28272834 .

[BaMaMo10] C. Balbuena, K. Marshall, and L. P. Montejano, On the connectivity and superconnected graphs with small diameter. Discrete Appl. Math. 58 (2010), 397403.

[BaSa12] C. Balbuena and J. Salas, A new bound for the connectivity of cages, Appl. Math. Letters 25 (2012) 1676-1680.

[BaTaMaLi09] C. Balbuena, J. Tang, K. Marshall, and Y. Lin, Superconnectivity of regular graphs with small diameter. Discrete Appl. Math. 157 (2009), 1349-1353. 
[BeCoHs95] J.-C. Bermond, F. Comellas, and D.F. Hsu, Distributed loop computer networks: a survey. J. Parallel Distrib. Comput. 24 (1995) 2-10.

[BeOePi02] L.W. Beineke, O. Oellermann, and R.E. Pippert. The average connectivity of a graph. Discrete Math. 252 (2002), 31-45.

[Bi71] N. Biggs, Intersection matrices for linear graphs, in: D.J.A. Welsh (Ed.), Combinatorial Mathematics and Its Applications, Academic Press, London, 1971, 15-23.

[Bi94] N. Biggs, Algebraic Graph Theory, Second edition, Cambridge University Press, Cambridge, 1994.

[BoFe70] F. Boesch and A.P. Felger, A class of non vulnerable graphs. Networks 2 (1970), 261-283.

[BoHaKa81] F. Boesch, F. Harary, and J.A. Kabell, Graphs as models of communication network vulnerability: Connectivity and persistence. Networks 11 (1981), 57-63.

[Bo79] B. Bollobás, On graphs with equal edge connectivity and minimum degree. Discrete Math. 28 (1979), 321-323.

[BoUeVo02] P. Bonsma, N. Ueffing, and L. Volkmann, Edge-cuts leaving components of order at least three. Discrete Math. 256 (2002), 431-439.

[BoKoZn68] J. Bosák, A. Kotzig, and S̆. Znám, Strongly geodetic graphs. J. Combin. Theory $\mathbf{5}$ (1968), 170-176.

[BoTi84] F. Boesch and R. Tindell, Circulants and their connectivities. J. Graph Theory 8 (1984), 487-499.

[Br95] A.E. Brouwer, Toughness and spectrum of a graph. Linear Algebra Appl. 226228 (1995), 267-271.

[Br96] A.E. Brouwer, Spectrum and connectivity of graphs. CWI Q. 9 (1996), 37-40.

[BrHa05] A.E. Brouwer and W.H. Haemers, Eigenvalues and perfect matchings. Linear Algebr. Appl. 395 (2005), 155-162.

[BrKo09] A.E. Brouwer and J.H. Koolen, The vertex-connectivity of a distance-regular graph. European J. Combin. 30 (2009), no. 3, 668-673.

[BrMe85] A.E. Brouwer and D.M. Mesner, The connectivity of strongly regular graphs. European J. Combin. 6 (1985), 215-216.

[CaFa99] A. Carmona and J. Fàbrega, On the superconnectivity and the conditional diameter of graphs and digraphs. Networks 34 (1999), 197-205.

[Ch66] G. Chartrand, A graph-theoretic approach to a communications problem. SIAM J. Appl. Math. 14 (1966), 778-781.

[Ch76] G. Chaty, On critically and minimally $k$ vertex (arc) strongly connected digraphs, Proc. Keszthely (1976), 193-203.

[ChLi02] E. Cheng and M.J. Lipman, Vulnerability issues of star graphs, alternating group graphs and split-stars: Strength and toughness. Discrete Appl. Math. 118 (2002), 163-179. 
[Co73] R.J. Cook, Heawood's theorem and connectivity. Mathematica Lond. 20 (1973), 201-207.

[CvDoSa95] D. Cvetković, M. Dragoš, and H. Sach, Spectra of Graphs, Theory and Applications. Third edition, J.A. Barth Verlag, Leipzig, 1995.

[DaRo99] M. Daven and C.A. Rodger, $(k, g)$-cages are 3-connected. Discrete Math. 199 (1999), 207-215.

[DaVo95] P. Dankelmann and L. Volkmann, New sufficient conditions for equality of minimum degree and edge-connectivity. Ars Comb. 40 (1995), 270-278.

[DaVo97] P. Dankelmann and L. Volkmann, Degree sequence conditions for maximally edge-connected graphs and digraphs. J. Graph Theory 26 (1997), 27-34.

[DaVo00] P. Dankelmann and L. Volkmann, Degree sequence conditions for maximally edge-connected graphs depending on the clique number. Discrete Math. 211 (2000), $217-223$.

[Es85] A.H. Esfahanian, Lower-bounds on the connectivities of a graph. J. Graph Theory 9 (1985), 503-511.

[Es88] A.H. Esfahanian and S.L. Hakimi, On computing a conditional edge-connectivity of a graph, Inf. Process. Lett. 27 (1988), 195-199.

[FaFi89] J. Fàbrega and M.A. Fiol, Maximally connected digraphs. J. Graph Theory 13 (1989), 657-668.

[FaFi94] J. Fàbrega and M.A. Fiol, Extraconnectivity of graphs with large girth. Discrete Math. 127 (1994), 163-170.

[FaFi96a] J. Fàbrega and M.A. Fiol, Bipartite graphs and digraphs with maximum connectivity. Discrete Appl. Math. 69 (1996), 269-277.

[FaFi96b] J. Fàbrega and M.A. Fiol, On the extraconnectivity of graphs. Discrete Math. 155 (1996), 49-57.

[Fi73] M. Fiedler, Algebraic connectivity of graphs. Czech. Math. J. 23 (1973), 298-305.

[Fi92] M.A. Fiol, On super-edge-connected digraphs and bipartite digraphs. J. Graph Theory 16 (1992), 545-555.

[Fi93] M.A. Fiol, The connectivity of large digraphs and graphs. J. Graph Theory 17 (1993), 31-45.

[Fi94] M.A. Fiol, The superconnectivity of large digraphs and graphs. Discrete Math. 124 (1994), 67-78.

[FiFa94] M.A. Fiol and J. Fàbrega, On the distance connectivity of graphs and digraphs. Discrete Math. 125 (1994), 169-176.

[FiFaEs90] M.A. Fiol, J. Fàbrega, and M. Escudero, Short paths and connectivity in graphs and digraphs. Ars Combin. 29B (1990), 17-31.

[FiGaYe97] M.A. Fiol, E. Garriga, and J.L.A. Yebra, The alternating polynomials and their relation with the spectra and conditional diameters of graphs. Discrete Appl. Math. 167/168 (1997), 297-307. 
[FiYeAl84] M.A. Fiol, J.L.A. Yebra, and I. Alegre, Line digraph iterations and the $(d, k)$ digraph problem. IEEE Trans. Comput. C-33 (1984), 400-403.

[FrOeSw00] G. Fricke, O.R. Oellermann, and H.C. Swart, The edge-connectivity, average edge-connectivity and degree conditions, manuscript (2000).

[FuHuRo97] H.L. Fu, K.C. Huang, and C.A. Rodgers, Connectivity of cages. J. Graph Theory 24 (1997), 187-191.

[Gh92] A. Ghafoor, Connectivity, persistence and fault diagnosis of interconnection networks based on $O_{k}$ and $2 O_{k}$ graphs. Discrete Appl. Math. 37/38 (1992), 205226.

[Go81] C. D. Godsil, Equiarboreal graphs. Combinatorica 1 (1981), 163-167.

[GoEn79] D.L. Goldsmith and R.C. Entringer, A sufficient condition for equality of edge-connectivity and minimum degree of a graph. J. Graph Theory 3 (1979), $251-255$.

[GoWh78] D.L. Goldsmith and A.T. White, On graphs with equal edge-connectivity and minimum degree. Discrete Math. 23 (1978), 31-36.

[GuWa86] M.A. Gurgel and Y. Wakabayashi, On k-leaf-connected graphs. J. Combin. Theory Ser. B 41 (1986), 1-16.

[Ha77] Y.O. Hamidoune, Sur les atomes d'un graphe orienté. C. R. Acad. Sci. Paris Sér. A 284 (1977), 1253-1256.

[Ha80] Y.O. Hamidoune, A property of $\alpha$-fragments of a digraph. Discrete Math. 31 (1980), 105-106.

[Ha81] Y.O. Hamidoune, Quelques problèmes de connexité dans les graphes orientés. J. Combin. Theory Ser. B 30 (1981), 1-10.

[Ha83] F. Harary, Conditional connectivity. Networks 13 (1983), 347-357.

[Ha84] Y.O. Hamidoune, On the connectivity of Cayley digraphs. European J. Combin. 5 (1984), 309-312.

[Ha89] Y.O. Hamidoune, Sur les atomes d'un graphe de Cayley infini. Discrete Math. 73 (1989), 297-300.

[Ha96] Y.O. Hamidoune, Additive group theory applied to network topology, pages 139 in D.-Z. Du et al. (Eds.), Combinatorial network theory, Appl. Optim. 1, Kluwer Academic Publishers, Dordrecht, 1996.

[HaLlSe92] Y.O. Hamidoune, A.S. Lladó, and O. Serra, The connectivity of hierarquical Cayley digraphs. Discrete Appl. Math. 37/38 (1992), 275-280.

[HaSe96] Y.O. Hamidoune and O. Serra, On small cuts separating Abelian Cayley graphs into two equal parts. Math. Syst. Theory 29 (1996), 407-409.

[HaHi99] S.G. Hartke and A.W. Higgins, Maximum degree growth of the iterated line graph. Electron. J. Combin. 6 (1999), Research paper 28.

[HeVo03a] A. Hellwig and L. Volkmann, Maximally edge-connected digraphs. Austral. J. Combin. 27 (2003), 23-32. 
[HeVo03b] A. Hellwig and L. Volkmann, Maximally local-edge-connected graphs and digraphs. Ars Combin. 72 (2004), 295-306.

[HeVo08a] A. Hellwig and L. Volkmann, The connectivity of a graph and its complement. Discrete Appl. Math. 156 (2008), 3325-3328.

[HeVo08b] A. Hellwig and L. Volkmann, Maximally edge-connected and vertexconnected graphs and digraphs: a survey. Discrete Math. 308 (2008), 3265-3296.

[ImSoOk85] M. Imase, T. Soneoka, and K. Okada, Connectivity of regular directed graphs with small diameter. IEEE Trans. Comput. C-34 (1985), 267-273.

[Im79] W. Imrich, On the connectivity of Cayley graphs. J. Combin. Theory Ser. B 26 (1979), 323-326.

[JiMu98] T. Jiang and D. Muyabi, Connectivity and separating sets of cages. J. Graph Theory 29 (1998), 35-44.

[Jo72] J.L. Jolivet, Sur la connexité des graphes orientés. C.R. Acad. Sci. Paris 274A (1972), 148-150.

[JuWa77] H.A. Jung and M.E. Watkins, On the connectivities of finite and infinite graphs. Mh. Math. 83 (1977), 121-131.

[Ka74] T. Kameda, Note on Halin's theorem on minimally connected graphs. J. Combin. Theory Ser. B 17 (1974), 1-4.

[Ke72] A.K. Kelmans, Asymptotic formulas for the probability of $k$-connectedness of random graphs. Theory Probab. Appl. 17 (1972), 243-254.

[KnNi03] M. Knor and L. Niepel, Connectivity of iterated line graphs. Discrete Appl. Math. 125 (2003), 255-266.

[Le74] L. Lesniak, Results on the edge-connectivity of graphs. Discrete Math. 8 (1974), $351-354$.

[LiMiBa05] Y. Lin, M. Miller, and C. Balbuena, Improved lower bound for the vertex connectivity of $(\delta ; g)$-cages. Discrete Math. 299 (2005), 162-171.

[LiMiBaMa06] Y. Lin, M. Miller, C. Balbuena, and X. Marcote, All $(k ; g)$-cages are edge-superconnected. Networks 47(2) (2006), 102-110.

[LiBaMaMi08] Y. Lin, C. Balbuena, X. Marcote, and M. Miller, On the connectivity of $(k, g)$-cages of even girth. Discrete Math. 308(15) (2008), 3249-3256.

[LuWuLuLi10] H. Lu, Y. Wu, Q. Lu, and Y. Lin, New improvements on connectivity of cages. Acta Math. Sinica English Series, 26(5) (2010), 1-12.

[Ma70] W. Mader, Über den Zusammenhang symmetrischer Graphen. Arch. Math. (Basel) 21 (1970), 331-336.

[Ma71a] W. Mader, Eine Eigenschaft der Atome endlicher Graphen. Arch. Math. (Basel) 22 (1971), 333-336.

[Ma71b] W. Mader, Minimale $n$-fach kantenzusammenhängende Graphen. Math. Ann. 191 (1971), 21-28. 
[MaBa04] X. Marcote and C. Balbuena, Edge-superconnectivity of cages. Networks 43(1) (2004), 54-59.

[MaBaPe04] X. Marcote, C. Balbuena, and I. Pelayo, Diameter, short paths and superconnectivity in digraphs. Discrete Math. 288 (2004), 113-123.

[MaBaPe07] X. Marcote, C. Balbuena, and I. Pelayo, On the connectivity of cages with girth five, six and eight. Discrete Math. 307 (2007), 1441-1446.

[MaBaPeFa05] X. Marcote, C. Balbuena, I. Pelayo, and J. Fàbrega, $(\delta, g)$-cages with $g \geq 10$ are 4-connected. Discrete Math. 301 (2005), 124-136.

[MaPeBa02] X. Marcote, I. Pelayo, and C. Balbuena, Every cubic cage is quasi 4connected. Discrete Math. 266 (2003), 311-320.

[PeBaGo01] I. Pelayo, C. Balbuena, and J. Gómez, On the connectivity of generalized p-cycles. Ars Combin. 58 (2001), 215-231.

[PeLaHe86] K. Peters, R. Laskar, and S. Hedetniemi, Maximal/minimal connectivity in graphs. Ars Comb. 21 (1986), 59-70.

[P175] J. Plesník, Critical graphs of given diameter. Acta Fac. Rerum Natur. Univ. Comenian. Math. 30 (1975), 71-93.

[PlZn74] J. Plesník and S̆. Znám, Strongly geodetic directed graphs. Acta Fac. Rerum Natur. Univ. Comenian., Math. Publ. 29 (1974), 29-34.

[PlZn89] J. Plesník and S̆. Znám, On equality of edge-connectivity and minimum degree of a graph. Arch. Math. (Brno) 25 (1989), 19-25.

[PluZh98] M. Plummer and X. Zha, On the connectivity of graphs embedded in surfaces. J. Combin. Theory Ser. B 72 (1998), 202-228.

[PluZh02] M. Plummer and X. Zha, On the connectivity of graphs embedded in surfaces II. Electron. J. Combin. 9 (2002), no. 1, Research Paper 38, 27 pp.

[ReKuHoLe82] S.M. Reddy, J.G. Kuhl, S.H. Hosseini, and H. Lee, On digraphs with minimum diameter and maximum connectivity, Proc. 20th Annual Allerton Conference (1982), 1018-1026.

[So92] T. Soneoka, Super edge-connectivity of dense digraphs and graphs. Discrete Appl. Math. 37/38 (1992), 511-523.

[SoNaIm85] T. Soneoka, H. Nakada, and M. Imase, Sufficient conditions for dense graphs to be maximally connected, Proc. ISCAS85 (1985), 811-814.

[SoNaImPe87] T. Soneoka, H. Nakada, M. Imase, and C. Peyrat, Sufficient conditions for maximally connected dense graphs. Discrete Math. 63 (1987), 53-66.

[ToVo93] J. Topp and L. Volkmann, Sufficient conditions for equality of connectivity and minimum degree of a graph. J. Graph Theory 17 (1993), 695-700.

[Vo88] L. Volkmann, Bemerkungen zum $p$-fachen Kantenzusammenhang von Graphen, [Remarks on the $p$-fold edge connectivity of graphs.] An. Univ. Bucure ti Mat. 37 (1988), 75-79. 
[Vo89] L. Volkmann, Edge-connectivity in p-partite graphs. J. Graph Theory 13 (1989), $1-6$.

[Vo03] L. Volkmann, Degree sequence conditions for equal edge-connectivity and minimum degree, depending on the clique number. J. Graph Theory 42 (2003), 234-245.

[WaXuWa03] P. Wang, B. Xu, and J. Wang, A note on the edge-connectivity of cages. Electron. J. Combin. 10 (2003), Note 2, 4 pp.

[Wa67] M.E. Watkins, A lower bound for the number of vertices of a graph. Amer. Math. Monthly 74 (1967), 297.

[Wa70] M.E. Watkins, Connectivity of transitive graphs. J. Combin. Theory 8 (1970), $23-29$.

[Wa02] M.E. Watkins, Personal communication, 2002.

[Wo73] D.R. Woodall, The binding number of a graph and its Anderson number. J. Combin. Theory Ser. B 15 (1973), 225-255.

[Xu92] J.-M. Xu, An inequality relating the order, maximum degree, diameter and connectivity of a strongly connected digraph. Acta Math. Appl. Sinica 8 (1992), 144-152.

[Xu94] J.-M. Xu, A sufficient condition for equality of arc-connectivity and minimum degree of a digraph. Discrete Math. 133 (1994), 315-318.

[XuWaWa02] B. Xu, P. Wang, and J. Wang, On the connectivity of $(4, g)$-cages. Ars Combin. 64 (2002), 181-192.

[XuXu02] Jun-Ming Xu and Ke-Li Xu, On restricted edge-connectivity of graphs. Discrete Math. 243 (2002), 291-298.

[ZhLi10] Z. Zhang and Q.H. Liu, Sufficient conditions for a graph to be $\lambda^{(k)}$-optimal with given girth and diameter. Networks 55 (2010), 119-124.

[ZhYu05] Z. Zhang and J.J. Yuan, A proof of an inequality concerning $k$-restricted edge connectivity. Discrete Math. 304 (2005), 128-134.

[ZhYu07] Z. Zhang and J.J. Yuan, Degree conditions for restricted-edge-connectivity and isoperimetric-edge-connectivity to be optimal. Discrete Math. 307 (2007), 293298. 